\title{
Duality Rotations and BPS Monopoles with Space and Time Noncommutativity
}

\author{
Paolo Aschieri \\ Sektion Physik der Ludwig-Maximilians-Universität \\ Theresienstr. 37, D-80333 München, Germany \\ e-mail address: aschieri@theorie.physik.uni-muenchen.de
}

\begin{abstract}
We show that noncommutative electromagnetism and Dirac-BornInfeld (DBI) theory with scalar fields are $S L(2, R)$ self-dual when noncommutativity is light-like and we are in the slowly varying field approximation. This follows from $S L(2, R)$ self-duality of the commutative DBI Lagrangian and of its zero slope limit that we study in detail.

We study a symmetry of noncommutative static configurations that maps spacenoncommutativity into space-time (and light-like) noncommutativity. $S L(2, R)$ duality is thus extended to space-noncommutativity. Via Seiberg-Witten map we study the nontrivial action of this symmetry on commutative DBI theory. In particular space-time noncommutative BPS magnetic monopoles corresponds to commutative BPS type magnetic monopoles with both electric and magnetic $B$-field background. Energy, charge and tension of these configurations are computed and found in agreement with that of a D1-string D3-brane system. We discuss the dual string-brane configuration.
\end{abstract}

Keywords: Duality; Born-Infeld; Noncommutative Gauge Theory; Monopoles

\section{Introduction}

Field theories on noncommutative spaces have received renewed interest since their appearance in M-theory and in string theory. In particular D-branes effective actions can be described by noncommutative gauge theories, the noncommutativity arising from a nonzero constant background NS $B$ field, see [17] and references therein. When $B \neq 0$ the effective physics on the D-brane can be described both by a commutative gauge theory $\mathcal{L}(F+B)$ and by a noncommutative one $\widehat{\mathcal{L}}(\widehat{F})$, where

$$
\widehat{F}_{\mu \nu}=\partial_{\mu} \widehat{A}_{\nu}-\partial_{\nu} \widehat{A}_{\mu}-i\left[\widehat{A}_{\mu} \star \widehat{A}_{\nu}\right]
$$

and $\star$ is Moyal star product; on coordinates $\left[x^{\mu} \star x^{\nu}\right]=x^{\mu} \star x^{\nu}-x^{\nu} \star x^{\nu}=i \Theta^{\mu \nu}$. The noncommutativity parameter $\Theta$ depends on $B$ and on the metric on the D- 
brane. The commutative/noncommutative descriptions are complementary and are related by Seiberg-Witten map (SW map). Initially space-noncommutativity has been considered $\left(\Theta^{i j} \neq 0\right.$ i.e. $\left.B_{i j} \neq 0\right)$ then theories also with time noncommutativity $\left(\Theta^{0 i} \neq 0\right.$ i.e $\left.B_{0 i} \neq 0\right)$ have been studied [20]. It turns out that unitarity of noncommutative Yang-Mills theory (NCYM) holds only if $\Theta$ is space-like or lightlike i.e. only if the electric and magnetic components of $\Theta$ (or $B$ ) are perpendicular and if the electric component is not bigger in magnitude than the magnetic component. These are precisely the NCYM theories that can be obtained from open strings in the decoupling limit $\alpha^{\prime} \rightarrow 0$ [23]. In this paper we consider these two kinds of space-time noncommutativity.

The effective D-brane actions, for slowly varying fields $(\partial F \sim 0)$ are respectively commutative and noncommutative Dirac-Born-Infeld $(\mathrm{DBI}): \mathcal{L}_{\mathrm{DBI}} \stackrel{\mathrm{SW} \text { map }}{\longleftrightarrow} \widehat{\mathcal{L}}_{\mathrm{DBI}}$. In the $\alpha^{\prime} \rightarrow 0$ limit [17] we obtain respectively a nonlinear commutative $U(1)$ gauge theory and noncommutative electromagnetism (NCEM); this last is the exact effective D-brane action for $\alpha^{\prime} \rightarrow 0$. Some D-brane properties are naturally studied in the noncommutative context e.g. T-duality 12, 14, instantons [13 and soliton solutions (in gauge theory and open string field theory) [18, [19]. Some other aspects turn out to be easier addressed in the context of commutative nonlinear electrodynamics; in particular, in 3+1 dimensions, electric-magnetic duality rotations. The general idea is to use SW map in order to get new insights about commutative/noncommutative DBI actions and D-brane physics.

In the first part of this paper we study duality rotations in the $\alpha^{\prime} \rightarrow 0$ limit and away from it, in both cases we see that noncommutative gauge theory is self-dual if $\Theta$ is light-like. $S L(2, R)$ self-duality of these theories is shown proving self-duality of the corresponding commutative actions. In particular we give, to all orders in $\Theta$, the explicit expression of the nonlinear $U(1)$ commutative theory that corresponds (for slowly varying fields) to NCEM. These results are also generalized to include a scalar field interacting with the gauge fields. We thus also provide new examples of Lagrangians satisfying Gaillard-Zumino self-duality condition. Formally (covariant derivatives, minimal couplings) NCEM resembles commutative $U(N)$ YM, and on tori with rational $\Theta$ the two theories are $T$-dual [14]. Self-duality of NCEM then hints to a possible duality symmetry of the equations of motion of $U(N)$ YM. Selfduality of NCEM was initially studied in 21] to first order in $\Theta$. In 42 pure NCEM with zero axion and dilaton has been seen to be self-dual under the $S O(2)$ compact subgroup of $S L(2, R)$. For $\Theta$ space-like as shown in 22 we do not have noncommutative gauge theory self-duality and the S-dual of space-like NCYM is a noncommutative open string theory decoupled from closed strings. Related work 
appeared in [24, 25, 26].

Since self-duality holds for $\Theta$ light-like, it may seem that this symmetry has a very restricted range of application. This is not the case. In the second half of this paper we show that for any space-noncommutative static solution we can turn on time-noncommutativity and obtain a static solution with light-like noncommutativity. We can then apply a duality rotation, switch off the time-noncommutativity and thus obtain a new solution of the original pure space-noncommutative theory. In particular we study BPS solutions of NCDBI and DBI theories with both electric and magnetic background thus answering the following questions: Consider the commutative BPS solution describing a D1-D3 system in the presence of a background magnetic field, what happens when we turn on a constant electric field? How is this configuration described in the noncommutative setting?

There has been quite some activity in the study of space-noncommutative BPS states and their relation, via SW map, to commutative BPS states, these studies have also tested SW map between commutative and space-noncommutative gauge theories [17, 31, 33, 34, 35, 36, 44]; in [32] pure time-noncommutativity is considered. Our work (with space-time noncommmutativity) fits in this context. In [29, 30] solutions to the BPS equations of NCEM with just space-noncommutativity are found, the solution in 29 describes a smeared monopole connected with a stringlike flux tube and is interpeted as a D1-string ending on a D3-brane with constant magnetic field background. In Section 3 we see that this solution remains a BPS solution also when we turn on time-noncommutativity. This is due to a symmetry of static configurations of NCDBI theory. Via SW map we obtain the nontrivial action of this symmetry in the corresponding commutative DBI theory and show that it is a rotation (boost) between the time component $A_{0}$ of the gauge potential and the worldvolume D-brane coordinates $x^{i}$. This boost, first studied in [10], is similar to the target space rotation that relates the linear monopole to the nonlinear monopole [33, 35, 10]. We show that this boost maps commutative BPS configurations into new BPS configurations; indeed these new configurations correspond via SW map to BPS configurations of NCEM, moreover they satisfy a first order differential equation and their energy equals a topological charge. In particular we describe explicitly the monopole plus string solution in a background that is both electric and magnetic. The monopole has the fundamental magneton charge and the string tension is that of a D1-string, this strongly suggests that we have a D1-D3 brane system with both electric and magnetic background. The D1-string tension is also matched with the string tension of the corresponding space-time noncommutative BPS monopole. We conclude briefly discussing the duality rotated string-brane 
configuration that describes an electric monopole in a magnetic background. For a different noncommutative approach to describe fundamental strings see [19].

\section{Duality rotations in (non)commutative gauge theory}

In this section we first review Gaillard-Zumino self-duality condition and self-duality of the (bosonic part of the IIB) D3-brane effective Lagrangian [5], 27]. We then present two simple arguments showing why duality rotations require $B$ and $\Theta$ lightlike in the zero slope limit. A necessary and sufficient condition for the zero slope limit to exist, independently form duality rotations, is given and we thus recover the condition for the existence of SW map. $S L(2, R)$ self-duality of NCDBI and NCEM is then discussed and in the last subsection the more general case of DBI theory with an extra scalar field (Higgs field) is studied. The zero slope of DBI Lagrangian is also explicitly given; when no scalar field is present this was given for $\Theta$ light-like in [42], recently it has independently appeared in a different (but for $d=4$ equivalent) form in 43$]$.

\subsection{Self-duality of D3-brane effective action}

Consider in four dimension and with (Einstein) metric $g_{E}$ the Lagrangian density (Lagrangian for short)

$$
\mathcal{L}\left(F_{\mu \nu}, g_{E \mu \nu}, \chi^{i}\right)=\sqrt{g_{E}} L\left(F_{\mu \nu}, g_{E \mu \nu}, \chi^{i}\right)
$$

where $\sqrt{g_{E}}=\sqrt{-\operatorname{det} g_{E}}$ and $\chi^{i}$ are some background fields that can possibly have space-time indices. If we define

$$
K^{* \mu \nu}=\frac{\partial L}{\partial F_{\mu \nu}} \quad\left(\frac{\partial F_{\rho \sigma}}{\partial F_{\mu \nu}}=\delta_{\rho}^{\mu} \delta_{\sigma}^{\nu}-\delta_{\rho}^{\nu} \delta_{\sigma}^{\mu}\right)
$$

then the Bianchi identity and the equations of motion (EOM) for $\mathcal{L}$ read

$$
\begin{aligned}
& \partial_{\mu}\left(\sqrt{g_{E}} F^{* \mu \nu}\right)=\partial_{\mu} \tilde{F}^{\mu \nu}=0, \\
& \partial_{\mu}\left(\sqrt{g_{E}} K^{* \mu \nu}\right)=\partial_{\mu} \tilde{K}^{\mu \nu}=0,
\end{aligned}
$$

\footnotetext{
a The Hodge dual of a two form $\Omega_{\mu \nu}$ is defined by $\Omega_{\mu \nu}^{*} \equiv \frac{1}{2} \sqrt{g_{E}} \epsilon_{\mu \nu \rho \sigma} \Omega^{\rho \sigma}$, where $\epsilon^{0123}=$ $-\epsilon_{0123}=1$ and $g_{E}$ has signature $(-,+,+,+)$. We have $\Omega_{\mu \nu}^{* *}=-\Omega_{\mu \nu}, \Omega^{* \mu \nu}=\frac{1}{2} \sqrt{g_{E}^{-1}} \epsilon^{\mu \nu \rho \sigma} \Omega_{\rho \sigma}$.
} 
where $\tilde{F}^{\mu \nu} \equiv \frac{1}{2} \epsilon^{\mu \nu \rho \sigma} F_{\rho \sigma}$ and $\tilde{K}^{\mu \nu} \equiv \frac{1}{2} \epsilon^{\mu \nu \rho \sigma} K_{\rho \sigma}$. Under the infinitesimal transformations

$$
\begin{aligned}
& \delta\left(\begin{array}{c}
K \\
F
\end{array}\right)=\left(\begin{array}{ll}
\mathrm{A} & \mathrm{B} \\
\mathrm{C} & \mathrm{D}
\end{array}\right)\left(\begin{array}{c}
K \\
F
\end{array}\right) \\
& \delta \chi^{i}=\xi^{i}(\chi),
\end{aligned}
$$

the $\mathcal{L}^{g_{E}, \chi}$ EOM (田) are mapped into the $\mathcal{L}^{g_{E}, \chi+\delta \chi}$ EOM. Consistency of (15), (16) with the definition of $K$, i.e. $\tilde{K}+\delta \tilde{K}=\frac{\partial}{\partial(F+\delta F)} \mathcal{L}\left(F+\delta F, g_{E}, \chi+\delta \chi\right)$ holds in particular if $\left(\begin{array}{l}\mathrm{AB} \\ \mathrm{CD}\end{array}\right)$ belongs to the Lie algebra of $S L(2, R)$, and the variation of the Lagrangian under (5),(河) can be written as

$$
\delta \mathcal{L} \equiv\left(\delta_{F}+\delta_{\chi}\right) \mathcal{L}=\frac{1}{4}(\mathrm{~B} F \tilde{F}+\mathrm{C} K \tilde{K})
$$

where $\delta_{F} \mathcal{L}=\frac{1}{2} \delta F \frac{\partial \mathcal{L}}{\partial F}, \delta_{\chi} \mathcal{L}=\delta \chi^{i} \frac{\partial \mathcal{L}}{\partial \chi^{i}}$ [3]. When (17) holds, under a finite $S L(2, R)$ rotation a solution $F$ of $\mathcal{L}^{g_{E}, \chi}$ is mapped into a solution $F^{\prime}$ of $\mathcal{L}^{g_{E}, \chi^{\prime}}$ and we say that $\mathcal{L}^{g_{E}, \chi}$ is self-dual; notice that the (Einstein) metric $g_{E}$ is invariant. A self-duality condition equivalent to ([) is obtained using (5) to evaluate the $\delta_{F} \mathcal{L}$ term in (7)

$$
\delta_{\chi} \mathcal{L}=\frac{1}{4} \mathrm{~B} F \tilde{F}-\frac{1}{4} \mathrm{C} K \tilde{K}-\frac{1}{2} \mathrm{D} F \tilde{K}
$$

If also the $\chi$ background fields are invariant under duality rotations then the maximal duality group is $U(1)$ [3], see also the nice review [28]. Viceversa we can always extend a $U(1)$ self-dual Lagrangian to a $S L(2, R)$ self-dual one introducing two real valued scalars $S=S_{1}+i S_{2}$ (axion and dilaton) [6, 7, 8]

Among the properties of self-dual theories we recall that $i$ ) self-duality implies invariance under Legendre transformations with respect to the field strength [8, 28]; ii) Consider a parameter or field $\lambda$ invariant under the transformations (5), (6) then, if $\xi^{i}$ is independent of $\lambda$, the derivative $\frac{\partial}{\partial \lambda} \mathcal{L}\left(F, g_{E}, \chi, \lambda\right)$ is also invariant under (5),(6) In particular since the metric is invariant then the energy momentum tensor is invariant.

Property ii) provides a way to construct new self-dual Lagrangians from known ones. Indeed given the self-dual lagrangian density $\mathcal{L}\left(F, \mathrm{~g}_{E}, \chi\right)$ consider the new lagrangian density

$$
\check{\mathcal{L}}\left(F, g_{E}+f(\phi), \chi\right) \equiv \mathcal{L}\left(F, g_{E}, \chi\right)
$$

where $g_{E \mu \nu}+f_{\mu \nu}\left(\phi_{E}\right)=\mathrm{g}_{E \mu \nu}$ and $f_{\mu \nu}\left(\phi_{E}\right)$ is a function of the metric $g_{E}$, the scalar fields $\phi_{E}^{a}$ and their derivatives e.g. $f_{\mu \nu}\left(\phi_{E}\right)=\sum_{a} \partial_{\mu} \phi_{E}^{a} \partial_{\nu} \phi_{E}^{a}$. Then $\check{\mathcal{L}}$ is self-dual

\footnotetext{
${ }^{b}$ Proof [3]: differentiate w.r.t. $\partial_{\lambda} \equiv \frac{\partial}{\partial \lambda}$ the expression $\delta \mathcal{L}=\delta_{\chi} \mathcal{L}+\delta_{F} \mathcal{L}=\delta_{\chi} \mathcal{L}+\frac{1}{2} \delta F \frac{\partial \mathcal{L}}{\partial F}$ to obtain $\partial_{\lambda} \delta \mathcal{L}=\delta_{\chi} \partial_{\lambda} \mathcal{L}+\frac{1}{2} \delta F \partial_{\lambda} \frac{\partial \mathcal{L}}{\partial F}+\frac{1}{2} \partial_{\lambda}(\delta F) \frac{\partial \mathcal{L}}{\partial F}=\delta \partial_{\lambda} \mathcal{L}+\frac{1}{2} \mathrm{C}\left(\partial_{\lambda} K\right) \tilde{K}$ i.e. $\delta \partial_{\lambda} \mathcal{L}=\partial_{\lambda}\left(\delta \mathcal{L}-\frac{1}{4} \mathrm{C} K \tilde{K}\right)=$ $\partial_{\lambda}\left(\delta \mathcal{L}-\frac{1}{4} \mathrm{C} K \tilde{K}-\frac{1}{4} \mathrm{~B} F \tilde{F}\right)=0$ where we used (7) in the last equality.
} 
under (5), (6) and $\delta g_{E}=\delta \phi_{E}^{a}=0$ indeed

$\left(\delta_{F}+\delta_{\chi}+\delta_{\phi_{E}}\right) \check{\mathcal{L}}=\left(\delta_{F}+\delta_{\chi}\right) \check{\mathcal{L}}=\left(\delta_{F}+\delta_{\chi}\right) \mathcal{L}=\frac{1}{4}(\mathrm{~B} F \tilde{F}+\mathrm{C} K \tilde{K})=\frac{1}{4}(\mathrm{~B} F \tilde{F}+\mathrm{C} \check{K} \tilde{\bar{K}})$

in the last line we used $\tilde{\tilde{K}}^{\mu \nu} \equiv \partial \check{\mathcal{L}} / \partial F_{\mu \nu}=\partial \mathcal{L} / \partial F_{\mu \nu}=\tilde{K}^{\mu \nu}$. Concerning the $\phi_{E}^{a}$ EOM

$$
\frac{\partial}{\partial \phi_{E}^{a}} \check{\mathcal{L}}-\partial_{\mu} \frac{\partial}{\partial \partial_{\mu} \phi_{E}^{a}} \check{\mathcal{L}}=0
$$

they do not change under the duality rotation because [recall $i i)] \phi_{E}^{a}$ is invariant and $\xi^{i}(\chi)$ is $\phi_{E}^{a}$ independent.

We now discuss self-duality of the D3-brane effective action in a IIB supergravity background with constant NS-NS two-form. The background two-form can be gauged away in the bulk and we are left with the field strength $\mathcal{F}=F+B$ on the D3-brane. Here $B$ is defined as the constant part of $\mathcal{F}$, or $\left.B \equiv \mathcal{F}\right|_{\text {spatial } \infty}$ since $F$ vanish at spatial infinity. For slowly varying fields the Lagrangian is a generalized version of Dirac-Born-Infeld Lagrangian, in string and in Einstein frames, it respectively reads:

$$
\begin{aligned}
\mathcal{L}_{\mathrm{DBI}} & =\frac{-1}{\alpha^{\prime 2} g_{s}} \sqrt{-\operatorname{det}\left(g+\alpha^{\prime} \mathcal{F}\right)}+\frac{1}{4} C \mathcal{F} \tilde{\mathcal{F}} \\
& =\frac{-1}{\alpha^{\prime 2}} \sqrt{-\operatorname{det}\left(g_{E}+\alpha^{\prime} S_{2}^{1 / 2} \mathcal{F}\right)}+\frac{1}{4} S_{1} \mathcal{F} \tilde{\mathcal{F}} \\
& =\frac{-1}{\alpha^{\prime 2}} \sqrt{-g_{E}} \sqrt{1+\frac{\alpha^{\prime 2}}{2} S_{2} \mathcal{F}^{2}-\frac{\alpha^{\prime}}{16} S_{2}^{2}\left(\mathcal{F} \mathcal{F}^{*}\right)^{2}}+\frac{1}{4} S_{1} \mathcal{F} \tilde{\mathcal{F}}
\end{aligned}
$$

where in the second line $g_{E}=g_{s}^{-\frac{1}{2}} g$ and $S=S_{1}+i S_{2}=C+\frac{i}{g_{s}}$ while, in the last line, we have simply expanded the $4 \mathrm{x} 4$ determinant and $\mathcal{F}^{2} \equiv \mathcal{F}_{\mu \nu} \mathcal{F}^{\mu \nu}, \mathcal{F F}^{*} \equiv$ $\mathcal{F}_{\mu \nu} \mathcal{F}^{* \mu \nu}$. Also, with abuse of notation, we have set $2 \pi \alpha^{\prime}=\alpha^{\prime}, 2 \pi g_{s}=g_{s}$.

Under the $S L(2, R)$ rotation

$$
\begin{gathered}
\left(\begin{array}{c}
\mathcal{K}^{\prime} \\
\mathcal{F}^{\prime}
\end{array}\right)=\left(\begin{array}{ll}
a & b \\
c & d
\end{array}\right)\left(\begin{array}{l}
\mathcal{K} \\
\mathcal{F}
\end{array}\right), \quad\left(\begin{array}{ll}
a & b \\
c & d
\end{array}\right) \in S L(2, R), \\
S^{\prime}=\frac{a S+b}{c S+d}, \quad g_{E}^{\prime}=g_{E}, \quad\left(\alpha^{\prime}\right)^{\prime}=\alpha^{\prime}
\end{gathered}
$$

where $\tilde{\mathcal{K}}=\frac{\partial}{\partial \mathcal{F}} \mathcal{L}$, the Lagrangian $\mathcal{L}_{\text {DBI }}$ satisfies the self-duality condition (8) with $F$ and $K$ replaced by $\mathcal{F}$ and $\mathcal{K}$. [The infinitesimal transformations (5),(6) can be obtained from the finite transformations (11), (12) using $\left.\left(\begin{array}{ll}a & b \\ c & d\end{array}\right) \approx\left(\begin{array}{ll}1 & 0 \\ 0 & 1\end{array}\right)+\left(\begin{array}{ll}A & B \\ C & D\end{array}\right)\right]$. 
For completeness we should add to $\mathcal{L}_{\mathrm{DBI}}$ the Wess-Zumino term $\tilde{C}_{4}=\frac{1}{24} \epsilon^{\mu \nu \rho \sigma} C_{\mu \nu \rho \sigma}$, where $C_{\mu \nu \rho \sigma}$ is the pull-back on the D3-brane of the RR four-form $C_{4}$. Under the $S L(2, R)$ action $C_{4}$ is invariant so that $\mathcal{L}_{\mathrm{D} 3}=\mathcal{L}_{\mathrm{DBI}}+\tilde{C}_{4}$ is self-dual. Moreover under $S L(2, R)$ the $g_{E}, S, C_{4}$ ten dimensional IIB supergravity background (with zero NS-NS and RR two-forms) is rotated into the $g_{E}, S^{\prime}, C_{4}$ one (with zero NS-NS and RR two-forms) 四, 27].

The explicit expression of $\mathcal{K}$ is

$$
\mathcal{K}_{\mu \nu}=\frac{S_{2} \mathcal{F}_{\mu \nu}^{*}+\frac{\alpha^{\prime 2}}{4} S_{2}^{2} \mathcal{F} \mathcal{F}^{*} \mathcal{F}_{\mu \nu}}{\sqrt{1+\frac{\alpha^{\prime 2}}{2} S_{2} \mathcal{F}^{2}-\frac{\alpha^{\prime}}{16} S_{2}^{2}\left(\mathcal{F} \mathcal{F}^{*}\right)^{2}}}+S_{1} \mathcal{F}_{\mu \nu} .
$$

Consider now a constant dilaton and axion $S$, then $S_{2}^{-1}=g_{s}=g_{\mathrm{YM}}^{2}$ plays the role of gauge coupling constant and the term $S_{1} \mathcal{F} \tilde{\mathcal{F}}$ becomes a total derivative that does not affect the $\mathcal{F}$ EOM (but is needed in $\mathcal{K}$ ). From (11), one can extract how $B$ (the value of $\mathcal{F}$ at spatial infinity) transforms

$$
B_{\mu \nu}^{\prime}=\left(c S_{1}+d\right) B_{\mu \nu}+c \frac{S_{2} B_{\mu \nu}^{*}+\frac{\alpha^{\prime 2}}{4} S_{2}^{2} B B^{*} B_{\mu \nu}}{\sqrt{1+\frac{\alpha^{\prime 2}}{2} S_{2} B^{2}-\frac{\alpha^{\prime}}{16} S_{2}^{2}\left(B B^{*}\right)^{2}}} ;
$$

this transformation is independent from the slowly varying fields approximation.

We have seen self-duality of $\mathcal{L}_{\mathrm{DBI}}$ and of $\mathcal{L}_{\mathrm{D} 3}$ under $\mathcal{F}, \mathcal{K}$ rotations. Let's now consider $F$, instead of $\mathcal{F}$, as the dynamical field in $\mathcal{L}_{\mathrm{DBI}}\left(F+B, g_{E}, S\right)$. This Lagrangian contains constant terms and terms linear in $F$ that are total derivatives, we drop them and obtain the Lagrangian

$$
\mathcal{L}_{\geq 2} \equiv \mathcal{L}_{\mathrm{DBI}}-\mathcal{L}_{0}-\mathcal{L}_{1} \equiv \mathcal{L}_{\mathrm{DBI}}-\left.\mathcal{L}_{\mathrm{DBI}}\right|_{F=0}-\left.\frac{1}{2} \frac{\partial \mathcal{L}_{\mathrm{DBI}}}{\partial F}\right|_{F=0} F
$$

that is at least quadratic in $F$. We also split $\tilde{\mathcal{K}}=\frac{\partial}{\partial \mathcal{F}} \mathcal{L}=\frac{\partial}{\partial F} \mathcal{L}$ as

$$
\tilde{\mathcal{K}}=\frac{\partial \mathcal{L}_{1}}{\partial F}+\tilde{K}, \quad \text { where } \tilde{K} \equiv \frac{\partial \mathcal{L}_{\geq 2}}{\partial F} .
$$

In order to show self-duality of $\mathcal{L}_{\geq 2}\left(F, g_{E}, B, S\right)$ w.r.t. $F$ we extract from (8) [i.e. $\left.\delta_{S} \mathcal{L}=\frac{1}{4} \mathrm{~B} \mathcal{F} \tilde{\mathcal{F}}-\frac{1}{4} \mathrm{C} \mathcal{K} \tilde{\mathcal{K}}-\frac{1}{2} \mathrm{D} \mathcal{F} \tilde{\mathcal{K}}\right]$ the terms that contains powers of $F$ of order $\geq 2$, and obtain

$$
\delta_{S} \mathcal{L}_{\geq 2}=\frac{1}{4} \mathrm{~B} F \tilde{F}-\frac{1}{4} \mathrm{C} K \tilde{K}-\frac{1}{2} \mathrm{D} F \tilde{K}-\frac{1}{2}\left(-\mathrm{C} \frac{\widetilde{\partial \mathcal{L}_{1}}}{\partial F}+\mathrm{D} B\right)\left(\tilde{K}-\left.\frac{1}{2} \frac{\partial \tilde{K}}{\partial F}\right|_{F=0} F\right) .
$$

We now disentangle in $\frac{\partial}{\partial F} \mathcal{L}_{\mathrm{DBI}}=\frac{\partial}{\partial B} \mathcal{L}_{\mathrm{DBI}}$ the terms of order 0 in $F$ from all the other terms and obtain

$$
\frac{\partial \mathcal{L}_{1}}{\partial F}=\frac{\partial \mathcal{L}_{0}}{\partial B} \quad \text { and } \quad \tilde{K}=\frac{\partial \mathcal{L}_{1}}{\partial B}+\frac{\partial \mathcal{L}_{\geq 2}}{\partial B}=\left.\frac{1}{2} \frac{\partial \tilde{K}}{\partial F}\right|_{F=0} F+\frac{\partial \mathcal{L}_{\geq 2}}{\partial B} .
$$

Relation (17) then becomes the self-duality equation for $\mathcal{L}_{\geq 2}$

$$
\left(\delta_{B}+\delta_{S}\right) \mathcal{L}_{\geq 2}=\frac{1}{4} \mathrm{~B} F \tilde{F}-\frac{1}{4} \mathrm{C} K \tilde{K}-\frac{1}{2} \mathrm{D} F \tilde{K} .
$$




\subsection{Open/closed strings and light-like noncommutativity}

The open and closed string parameters are related by (see [17], the expressions for $G$ and $\Theta$ first appeared in [15])

$$
\begin{aligned}
& \frac{1}{g+\alpha^{\prime} B}=G^{-1}+\frac{\Theta}{\alpha^{\prime}} \\
& g^{-1}=\left(G^{-1}-\Theta / \alpha^{\prime}\right) G\left(G^{-1}+\Theta / \alpha^{\prime}\right)=G^{-1}-\alpha^{\prime-2} \Theta G \Theta \\
& \alpha^{\prime} B=-\left(G^{-1}-\Theta / \alpha^{\prime}\right) \Theta / \alpha^{\prime}\left(G^{-1}+\Theta / \alpha^{\prime}\right) \\
& G_{s}=g_{s} \sqrt{\frac{\operatorname{det} G}{\operatorname{det}\left(g+\alpha^{\prime} B\right)}}=g_{s} \sqrt{\operatorname{det} G \operatorname{det}\left(G^{-1}+\Theta / \alpha^{\prime}\right)}=g_{s} \sqrt{\operatorname{det} g^{-1} \operatorname{det}\left(g+\alpha^{\prime} B\right)}
\end{aligned}
$$

If we define $\Omega \equiv-G \frac{\Theta}{\alpha^{\prime 2}} G$ then $g, B$ are related to $G, \Omega$ via the change of coordinates

$$
\begin{aligned}
& G=M^{T} g M \\
& \Omega=M^{T} B M
\end{aligned} \quad \text { with } \quad M=g^{-1}\left(g+\alpha^{\prime} B\right)=G^{-1}\left(G+\alpha^{\prime} \Omega\right)=\left(G^{-1}-\Theta / \alpha^{\prime}\right) G .
$$

The decoupling limit $\alpha^{\prime} \rightarrow 0$ with $G_{s}, G, \Theta$ nonzero and finite [17] leads to a well defined field theory only if $B$ is space-like or light-like [23]. Looking at the closed and open string coupling constants it is easy to see why one needs this space-like or lightlike condition on $B$. Consider the coupling constants ratio $G_{s} / g_{s}$, that expanding the $4 \mathrm{x} 4$ determinant reads (here $B^{2}=B_{\mu \nu} B_{\rho \sigma} g^{\mu \rho} g^{\nu \sigma}, \Theta^{2}=\Theta^{\mu \nu} \Theta^{\rho \sigma} G_{\mu \rho} G_{\nu \sigma}$ and so on)

$$
\frac{G_{s}}{g_{s}}=\sqrt{1+\frac{\alpha^{\prime-2}}{2} \Theta^{2}-\frac{\alpha^{\prime-4}}{16}\left(\Theta \Theta^{*}\right)^{2}}=\sqrt{1+\frac{\alpha^{\prime 2}}{2} B^{2}-\frac{\alpha^{\prime 4}}{16}\left(B B^{*}\right)^{2}} .
$$

Both $G_{s}$ and $g_{s}$ must be positive; since $G$ and $\Theta$ are by definition finite for $\alpha^{\prime} \rightarrow 0$ this implies $\Theta \Theta^{*}=0$ and $\Theta^{2} \geq 0$. Now $\Theta \Theta^{*}=0 \Leftrightarrow \operatorname{det} \Theta=0 \Leftrightarrow \operatorname{det} B=0 \Leftrightarrow$ $B B^{*}=0$. In this case from (21) we also have $\Theta^{2}=\alpha^{\prime 4} B^{2}$. In conclusion a necessary condition for the $\alpha^{\prime} \rightarrow 0$ limit to be well defined is

$$
B^{2} \geq 0, \quad B B^{*}=0 \quad \text { i.e. } \quad \Theta^{2} \geq 0, \quad \Theta \Theta^{*}=0 \text {. }
$$

This is the condition for $B$ (and $\Theta$ ) to be space-like or light-like. Indeed with Minkowski metric (22) reads $\vec{B}^{2}-\vec{E}^{2} \geq 0$ and $\vec{E} \perp \vec{B}$. In the next subsection we see that a necessary and sufficient condition for the $\alpha^{\prime} \rightarrow 0$ limit to be well defined is (22) and

$$
1-\frac{1}{2} \Theta F \neq 0
$$

Notice that space time is divided in the regions $1-\frac{1}{2} \Theta F>0$ and $1-\frac{1}{2} \Theta F<0$. We generically have $1-\frac{1}{2} \Theta F>0$ because we have considered $F=0$ at spatial infinity. 
Condition (23) is the condition for SW map to be well defined [17, see also [40, indeed for constant $F$ it is equivalent to the existence of $\widehat{F}_{\mu \nu}=\left[\frac{1}{1+F \Theta} F\right]_{\mu \nu}$.

We now require the $\alpha^{\prime} \rightarrow 0$ limit to be compatible with duality rotations. From (22) we immediately see that we have to consider only the light-like case $B^{2}=$ $B B^{*}=0$; indeed under $S L(2, R)$ rotations the electric and magnetic fields mix up; in particular under an $S L(2, R)$ rotation $\left(\begin{array}{ll}a & b \\ c & d\end{array}\right)$ with $d=-c S_{1}$, a space-like $B$ becomes time-like.

A complementary perspective that leads to the light-like condition $B^{2}=B B^{*}=$ 0 is as follows. We fix $G_{s}, G, \Theta$ and rescale $\alpha^{\prime} \rightarrow \epsilon \alpha^{\prime}$. This defines the $\epsilon$ dependence of the open string variables: $g_{s}(\epsilon), g(\epsilon), B(\epsilon)$. Under a duality rotation we obtain $g_{s}^{\prime}(\epsilon), g^{\prime}(\epsilon), B^{\prime}(\epsilon)$. The two operations of duality rotation and $\alpha^{\prime}$ rescaling are compatible if they commute, i.e. the open string variables $G_{s}^{\prime}, G^{\prime}, \Theta^{\prime}$ obtained from $g_{s}^{\prime}(\epsilon), g^{\prime}(\epsilon), B^{\prime}(\epsilon)$ must be $\epsilon$ independent. This is the case if $\Theta$ (or $B$ ) is light-like. As shown in 22 if $B$ is space-like and $S_{1}=0$, under a $\pi / 2$ rotation $G^{\prime}$ depends linearly on $\epsilon^{-1}$; letting $\epsilon \rightarrow 0$ open strings decouple from closed strings but massive open string states do not decouple from massless open string states so that the S-dual of this NCEM is a noncommutative open string theory.

\subsection{Self-duality of NCDBI and NCEM}

We now study duality rotations for noncommutative Dirac-Born-Infeld (NCDBI) theory and its zero slope limit that is noncommutative electromagnetism (NCEM). The relation between NCDBI and DBI Lagrangians is 17

$$
\widehat{\mathcal{L}}_{\mathrm{DBI}}\left(\widehat{F}, G, \Theta, G_{s}, C\right)=\mathcal{L}_{\mathrm{DBI}}\left(F+B, g, g_{s}, C\right)+O(\partial F)+\text { tot.der. }
$$

where $\widehat{F}$ is the noncommutative $U(1)$ field strength and $O(\partial F)$ stands for higher order derivative corrections (these corrections are at least quadratic in $\partial$ ). The NCDBI Lagrangian is:

$$
\widehat{\mathcal{L}}_{\mathrm{DBI}}\left(\widehat{F}, G, \Theta, G_{s}, C\right)=\frac{-1}{\alpha^{\prime 2} G_{s}} \sqrt{-\operatorname{det}\left(G+\alpha^{\prime} \widehat{F}\right)}+\frac{1}{4} C \widehat{F} \widetilde{F}+O(\partial \widehat{F}) ;
$$

as in the commutative case also $C \widehat{F} \widetilde{F}$ is a total derivative.

In the slowly varying field approximation the action of duality rotations on $\widehat{\mathcal{L}}_{\mathrm{DBI}}$ is derived from self-duality of $\mathcal{L}_{\mathrm{DBI}}$. If $\widehat{F}$ is a solution of the $\widehat{\mathcal{L}}_{\mathrm{DBI}}^{G, \Theta, G_{s}}$ EOM then $\widehat{F}^{\prime}$ obtained via $\widehat{F} \stackrel{\mathrm{SW} \text { map }}{\longleftrightarrow} \mathcal{F} \stackrel{\text { duality rot. }}{\longleftrightarrow} \mathcal{F}^{\prime} \stackrel{\mathrm{SW} \text { map }}{\longleftrightarrow} \widehat{F}^{\prime}$ is a solution of the $\widehat{\mathcal{L}}_{\mathrm{DBI}}^{G^{\prime}, \Theta^{\prime}, G_{s}^{\prime}}$ EOM where $G^{\prime}, \Theta^{\prime}, G_{s}^{\prime}$ are obtained using (19) from $g^{\prime}=S_{2}^{\prime-\frac{1}{2}} g_{E}$ and $B^{\prime}$. More precisely in (24) we can replace $\mathcal{L}_{\mathrm{DBI}}\left(F+B, g_{E}, S\right)$ with (15) that as shown in (18) 
is self-dual. Using

$$
\frac{1}{g_{s}} \sqrt{-\operatorname{det}\left(g+\alpha^{\prime} \mathcal{F}\right)}=\frac{\sqrt{G}}{G_{s}} \sqrt{\frac{\operatorname{det}\left(g+\alpha^{\prime} B+\alpha^{\prime} F\right)}{\operatorname{det}\left(g+\alpha^{\prime} B\right)}}=\frac{1}{G_{s}} \sqrt{-\operatorname{det}\left(G+\alpha^{\prime} F+G \Theta F\right)}
$$

we then rewite $\mathcal{L}_{\geq 2}\left(F, g_{E}, B, S\right)$ in terms of the open string parameters $G, \Theta, G_{s}, C$

$$
\mathcal{L}_{\geq 2}\left(F, G, \Theta, G_{s}, C\right)=\frac{-1}{\alpha^{\prime 2} G_{s}} \sqrt{-\operatorname{det}\left(G+\alpha^{\prime} F+G \Theta F\right)}+\frac{\sqrt{G}}{\alpha^{\prime 2} G_{s}}\left(1-\frac{1}{2} \Theta F\right)
$$

This lagrangian satisfies the self-duality condition (प) with $\delta \mathcal{L}_{\geq 2}=\left(\delta_{F}+\delta_{G}+\delta_{\Theta}+\right.$ $\left.\delta_{G_{s}}+\delta_{C}\right) \mathcal{L}_{\geq 2}$. Via $\widehat{F} \stackrel{\text { SW map }}{\longleftrightarrow} F \stackrel{\text { duality rot. }}{\longleftrightarrow} F^{\prime} \stackrel{\text { SW map }}{\longleftrightarrow} \widehat{F}^{\prime}$ we then map solutions $\widehat{F}$ of the $\widehat{\mathcal{L}}_{\mathrm{DBI}}^{G, \Theta, G_{s}}$ EOM to solutions $\widehat{F}^{\prime}$ of the $\widehat{\mathcal{L}}_{\mathrm{DBI}}^{G^{\prime}, \Theta^{\prime}, G_{s}^{\prime}}$ EOM. (The non uniqueness of SW map is not an issue here because SW map is unique up to gauge transformations).

In the light-like case relations (19) simplify considerably. The open and closed string coupling constants coincide so that $S=S_{1}+i S_{2}=C+\frac{i}{g_{s}}=C+\frac{i}{G_{s}}$. Use of the relations

$$
\Omega_{\mu \rho}^{*} \Omega^{* \rho \nu}-\Omega_{\mu \rho} \Omega^{\rho \nu}=\frac{1}{2} \Omega^{2} \delta_{\mu}^{\nu}, \quad \Omega_{\mu \rho} \Omega^{* \rho \nu}=\Omega^{*}{ }_{\mu \rho} \Omega^{\rho \nu}=\frac{-1}{4} \Omega \Omega^{*} \delta_{\mu}{ }^{\nu}
$$

valid for any antisymmetric tensor $\Omega$, shows that any two-tensor at least cubic in $\Theta$ (or $B$ ) vanishes. It follows that $g^{-1} G \Theta=\Theta$ and that the raising or lowering of the $\Theta$ and $B$ indices is independent from the metric used. We also have

$$
B_{\mu \nu}=-\alpha^{\prime-2} \Theta_{\mu \nu} \quad \text { (in string frame) }
$$

In the light-like case the $B$ rotation (14) simplifies to

$$
B_{\mu \nu}^{\prime}=\left(c S_{1}+d\right) B_{\mu \nu}+c S_{2} B_{\mu \nu}^{*} .
$$

[we do not specify whether $B^{*}{ }_{\mu \nu}\left(\right.$ or $\Theta^{* \mu \nu}$ ) is obtained with Einstein or string metric because $B^{*}{ }_{\mu \nu}$ (or $\Theta^{* \mu \nu}$ ) is the same in both metrics]. In order to describe the $S L(2, R)$ action on the open string variables we express them in Einstein frame and then use (12) and (30) to obtain

$$
G_{E}^{\prime}=G_{E} \quad, \quad \Theta^{\prime \mu \nu}=\frac{\left(c S_{1}+d\right) \Theta^{\mu \nu}+c S_{2} \Theta^{* \mu \nu}}{|c S+d|^{2}} \quad \text { i.e. } \quad \Theta^{\prime}+i \Theta^{\prime *}=\frac{\Theta+i \Theta^{*}}{c S+d}
$$

Therefore, for $\Theta$ light-like, $G_{E}$ is invariant under duality rotations, (27) is self-dual and NCDBI Lagrangian is self-dual. In particular if $S_{1}=0$, under a $\pm \pi / 2$ rotation $S_{2} \rightarrow-\frac{1}{S_{2}}$ and $\Theta \rightarrow \pm \frac{1}{S_{2}} \Theta^{*}$.

We now consider the decoupling limit. The determinant in (26) can be evaluated as sum of products of traces (Newton-Leverrier formula). Each trace can then be 
rewritten in terms of the six basic Lorentz invariants $F^{2}, F F^{*}, F \Theta, F \Theta^{*}, \Theta^{2}$, and $\Theta \Theta^{*}=0$ because of (22). Explicitly

$$
\begin{aligned}
& \operatorname{det} G^{-1} \operatorname{det}(G+\left.\alpha^{\prime} F+G \Theta F\right)= \\
&\left(1-\frac{1}{2} \Theta F\right)^{2}+\alpha^{\prime 2}\left[\frac{1}{2} F^{2}+\frac{1}{4} \Theta F^{*} F F^{*}-\frac{1}{32} \Theta^{2}\left(F F^{*}\right)^{2}\right]-\alpha^{\prime 4}\left(\frac{1}{4} F F^{*}\right)^{2}
\end{aligned}
$$

Finally we take the $\alpha^{\prime} \rightarrow 0$ limit of (27). This limit is well defined iff $1-\frac{1}{2} \Theta F>0$, if $1-\frac{1}{2} \Theta F<0$ we obtain also an infinite total derivative addend that we can neglect; with this prescription the $\alpha^{\prime} \rightarrow 0$ limit is well defined iff $1-\frac{1}{2} \Theta F \neq 0$. We denote by $\mathcal{L}_{\alpha^{\prime} \rightarrow 0}$ the resulting Lagrangian, in string and Einstein frame we respectively have

$$
\begin{aligned}
\mathcal{L}_{\alpha^{\prime} \rightarrow 0} & =\frac{\sqrt{G}}{G_{s}} \frac{-\frac{1}{4} F^{2}-\frac{1}{8} \Theta F^{*} F F^{*}+\frac{1}{64} \Theta^{2}\left(F F^{*}\right)^{2}}{\left|1-\frac{1}{2} \Theta F\right|}+\frac{1}{4} C F \tilde{F} \\
& =\sqrt{G_{E}} S_{2} \frac{-\frac{1}{4} F^{2}-\frac{1}{8} \Theta F^{*} F F^{*}+\frac{1}{64} \Theta^{2}\left(F F^{*}\right)^{2}}{\left|1-\frac{1}{2} \Theta F\right|}+\frac{1}{4} S_{1} F \tilde{F} .
\end{aligned}
$$

We thus have an expression for NCEM in terms of $G, \Theta, G_{s}, C$ and $F$

$$
\begin{aligned}
\widehat{\mathcal{L}}_{\mathrm{EM}} & \equiv \frac{\sqrt{G}}{G_{s}} \frac{-1}{4} \widehat{F}^{2}+\frac{1}{4} C F \tilde{F} \\
& =\mathcal{L}_{\alpha^{\prime} \rightarrow 0}+O(\partial F)+\text { tot. der. }
\end{aligned}
$$

In the light-like case we have $\Theta^{2}=0$ and one can directly verify that the Lagrangian (32) when $1-\frac{1}{2} \Theta F>0$ satisfies the self-duality condition (8) with $\chi=\Theta, S$ so that (in the slowly varying field approximation) NCEM is self-dual. More easily, self-duality of (32) when $1-\frac{1}{2} \Theta F>0$ follows from self-duality of $\mathcal{L}_{\geq 2}\left(F, G, \Theta, G_{s}, C\right)$; indeed, for $\Theta$ light-like we have seen that the $\alpha^{\prime} \rightarrow 0$ limit is well defined and compatible with duality rotations 9 .

\footnotetext{
${ }^{\text {cSelf-duality in the region } 1-\frac{1}{2} \Theta F}<0$ holds too. Because of the absolute value the first addend in the Lagrangian gains a minus sign. This new Lagrangian satisfies the self-duality condition (8) under the $S L(2, R)$ rotation (19), (12), $G_{E}^{\prime}=G_{E}$ and $\Theta^{\prime}+i \Theta^{\prime *}=\frac{\Theta+i \Theta^{*}}{c \bar{S}+d}$. Proof:

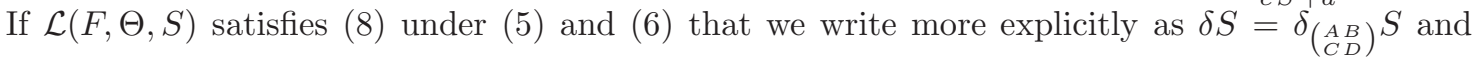
$\delta \Theta=\delta_{\left(\begin{array}{c}A B \\ C D\end{array}\right) ; S} \Theta$, then $-\mathcal{L}(F, \Theta, S)$ satisfies (8) under $\left(\right.$ 可 and $\delta_{\left(\begin{array}{c}A-B \\ -C\end{array}\right)} S$ and $\delta_{\left(\begin{array}{c}A-B \\ -C D\end{array}\right) ; S} \Theta$. Similarly for $-\mathcal{L}(F, \Theta,-\bar{S})$. Finally notice that $\delta_{\left(\begin{array}{c}A-B \\ -C\end{array}\right)}(-\bar{S})$ is equivalent to $\delta_{\left(\begin{array}{c}A B \\ C\end{array}\right)} S$. About the new $\Theta$ transformation rule we recall that the original $\Theta$ transformation was found setting $F=0$ i.e. was found in the region $1-\frac{1}{2} \Theta F>0$, there is no reason why it should hold also in the $1-\frac{1}{2} \Theta F<0$ region.
} 


\subsection{Dirac-Born-Infeld theory with extra scalar fields}

The four dimensional DBI Lagrangian with scalar fields (Higgs fields) $\phi^{a}, a=1, \ldots n$, can be obtained from the $4+n$ dimensional pure DBI Lagrangian where the gauge fields $A_{M}=\left(A_{\mu}, A_{a}\right)$ depend only on the $x^{\mu}$ coordinates $\mu=0, \ldots 3$, the metric $g_{M N}$ satisfies $g_{\mu b}=0, g_{a b}=\delta_{a b}$, the $B_{M N}$ field satisfies $B_{\mu b}=B_{a b}=0$ and $\phi_{a}=\alpha^{\prime} A_{a}$ [10].

This derivation applies also in the noncommutative case, here $G_{M N}$ satisfies $G_{\mu b}=0, G_{a b}=\delta_{a b}$. Relation (19) then implies $\Theta^{\mu b}=\Theta^{a b}=0$. We also set $\widehat{\phi}_{a}=\alpha^{\prime} \widehat{A}_{a}, \hat{D}_{\mu} \widehat{\phi}_{a}=\partial_{\mu} \widehat{\phi}_{a}-i\left[\widehat{A}_{\mu} \star \widehat{\phi}_{a}\right]$ and consider $\left[\widehat{\phi}_{a} \star \widehat{\phi}_{b}\right]=0$. The fields $\widehat{A}_{\mu}, \widehat{\phi}_{a}$ are related to the $A_{\mu}, \phi^{a}$ fields via the $4+n$ dimensional SW map. We have

$$
\begin{aligned}
\mathcal{L}_{\mathrm{DBI}}\left(\mathcal{F}, \phi, g, g_{s}, C\right)= & \frac{-1}{\alpha^{\prime 2} g_{s}} \sqrt{-\operatorname{det}\left(g+\alpha^{\prime} \mathcal{F}+\alpha^{\prime 2} \partial \phi_{a} \partial \phi^{a}\right)}+\frac{1}{4} C \mathcal{F} \tilde{\mathcal{F}} \\
\widehat{\mathcal{L}}_{\mathrm{DBI}}\left(\widehat{F}, \phi, G, \Theta, G_{s}, C\right)= & \mathcal{L}_{\mathrm{DBI}}\left(F+B, \phi, g, g_{s}, C\right)+O(\partial F, \partial \partial \phi)+\text { tot.der. } \\
\widehat{\mathcal{L}}_{\mathrm{DBI}}\left(\widehat{F}, \widehat{\phi}, G, \Theta, G_{s}, C\right)= & \frac{-1}{\alpha^{\prime 2} G_{s}} \sqrt{-\operatorname{det}\left(G+\alpha^{\prime} \widehat{F}+\alpha^{\prime 2} \hat{D} \widehat{\phi} \hat{\phi} \hat{D} \widehat{\phi}^{a}\right)}+\frac{1}{4} C \widehat{F} \widetilde{\widehat{F}} \\
& +O(\partial \widehat{F}, \partial \hat{D} \widehat{\phi})
\end{aligned}
$$

Since (34) with $\phi^{a}=g_{s}^{\frac{1}{4}} \phi_{E}^{a}$ can also be obtained from four dimensional DBI with metric $\mathrm{g}_{E}=g_{E}+\partial \phi_{a_{E}} \partial \phi_{E}^{a}$ as in (9), we have that (34) is self-dual under the $S L(2, R)$ action $\phi_{E}^{a}{ }^{\prime}=\phi_{E}$ and (12). We also define $\mathcal{L}_{\geq 2}$ as in (15) provided that each time we set $F=0$ we also set $\phi^{a}=0 . \mathcal{L}_{\geq 2}$ differs from (34) just by a constant term and a total derivative term. Formula (17) is then obtained from the self-duality condition for $\mathcal{L}_{\mathrm{DBI}}\left(F+B, \phi, g, g_{s}, C\right)$ discarding the terms that do not contain $\phi^{a}$ and have order 0 or 1 in $F$. As in (18) we conclude that $\mathcal{L}_{\geq 2}\left(F, \phi_{E}, g_{E}, B, S\right)$ is self-dual w.r.t. $F$. We now rewrite $\mathcal{L}_{\geq 2}$ as $\mathcal{L}_{\geq 2}\left(F, \phi, G, \Theta, G_{s}, C\right)$; for $\theta$ light-like $\mathcal{L}_{\geq 2}\left(F, \phi, G, \Theta, G_{s}, C\right)$ is self-dual under $\phi_{E}^{a}{ }^{\prime}=\phi_{E}^{a}$ and (31) so that (36) is self-dual.

In the $\alpha^{\prime} \rightarrow 0$ limit, (36) gives noncommutative electromagnetism coupled to Higgs fields

$$
\begin{aligned}
\widehat{\mathcal{L}} & \equiv \frac{\sqrt{G}}{G_{s}}\left(\frac{-1}{4} \widehat{F}^{2}-\frac{1}{2} \hat{D} \widehat{\phi}_{a} \hat{D} \widehat{\phi}^{a}\right)+\frac{1}{4} C F \tilde{F} \\
& =\mathcal{L}_{\alpha^{\prime} \rightarrow 0}+O(\partial F)+\text { tot. der. }
\end{aligned}
$$

where the equality in the last line is the $\alpha \rightarrow 0$ limit of (35), and where now $\mathcal{L}_{\alpha^{\prime} \rightarrow 0}$ with $\Theta$ light-like and $1-\frac{1}{2} \Theta F>0$ is given by

$$
\mathcal{L}_{\alpha \hookrightarrow 0}=\frac{\sqrt{G}}{G_{s}} \frac{-\frac{1}{4} F^{2}-\frac{1}{8} \Theta F^{*} F F^{*}-\frac{1}{2} \operatorname{tr}\left(\partial \phi_{a} \Theta F F \Theta \partial \phi^{a}\right)}{1-\frac{1}{2} \Theta F}
$$




$$
+\frac{\sqrt{G}}{G_{s}}\left[\operatorname{tr}\left(\partial \phi_{a} F \Theta \partial \phi^{a}\right)-\frac{1}{2}\left(1-\frac{1}{2} \Theta F\right) \partial \phi_{a} \partial \phi^{a}\right]+\frac{1}{4} C F \tilde{F} .
$$

Compatibility of the decoupling limit with duality rotations implies that this Lagrangian is self-dual under $\phi_{E}^{a \prime}=\phi_{E}^{a}$ and (31). We conclude that (in the slowly varying field approximation) NCEM with scalar fields is self-dual.

We end this section observing that the change $\Theta \rightarrow \Theta^{\prime}$ under a duality rotation, can be cancelled by a rotation in space and a $\Theta$ rescaling, or also by a Lorentz rotation in space-time.

\section{Gauge theory with space-time noncommutativity}

We have seen that self-duality of NCDBI and NCEM requires light-like noncommutativity. In this section we study a symmetry of static solutions of noncommutative Lagrangians, we call it $\theta-\theta^{\varepsilon}$ symmetry. To any space-noncommutative solution we associate a space-time, and in particular light-like, noncommutative solution and viceversa. In NCDBI and NCEM noncommutative self-duality acts also within space-noncommutativity, it is a duality rotation obtained first going to light-like noncommutativity, then applying a duality rotation and finally going back to space noncommutativity. In Subsection 3.2 and 3.3 we study how the $\theta-\theta^{\varepsilon}$ symmetry of NCDBI acts on the commutative DBI theory. New BPS type solutions of DBI with both electric and magnetic backgrounds, and their corresponding noncommutative solutions, are presented in Subsection 3.5.

We use the following notations: $\Theta$ is a generic constant noncommutativity tensor, we have $\left[x^{\mu} \star x^{\nu}\right]=i \Theta^{\mu \nu} ; \theta$ is just a space-noncommutativity tensor $\theta^{i j}, \theta^{0 i}=0$; $\theta^{\varepsilon}$ is a space-time noncommutativity tensor obtained from $\theta$ adding electric components, $\theta^{\varepsilon i j}=\theta^{i j}, \theta^{\varepsilon 0 i}$. In three vector notation the electric and magnetic components of $\theta$, respectively $\theta^{\varepsilon}$, are $(0, \boldsymbol{\theta})$ and $(\boldsymbol{\varepsilon}, \boldsymbol{\theta})$. The background fields corresponding to $\Theta, \theta, \theta^{\varepsilon}$ are $B, b, b^{\varepsilon}$. We also recall that we have set $2 \pi \alpha^{\prime}=\alpha^{\prime}, 2 \pi g_{s}=g_{s}$.

\subsection{Static solutions of space-time noncommutative gauge theory and SW map}

Consider a noncommutative Lagrangian $\widehat{\mathcal{L}}^{\theta}=\widehat{\mathcal{L}}\left(\widehat{F}, \widehat{\phi}, G, \star_{\theta}\right)$. The EOM for $\widehat{\mathcal{L}}^{\theta}$ read

$$
f_{\alpha}\left(\widehat{F}, \widehat{\phi}, G, \star_{\theta}\right)=0
$$


where $f_{\alpha}$ are functions of the noncommutative fields and their derivatives. We notice that a static solution of the $\widehat{\mathcal{L}}^{\theta}$ EOM (39) is also a static solution of (39) with $\theta^{\varepsilon}$ instead of $\theta$, i.e. it is a static solution of the $\widehat{\mathcal{L}}^{\theta^{\varepsilon}}$ EOM. Indeed the star products $\star_{\theta}$ and $\star_{\theta^{\varepsilon}}$ act in the same way on time independent fields. Also the energy and charges of the solution are invariant. A similar symmetry property holds if the fields are independent from a coordinate $x^{\mu}$ (not necessarily $t$ ). This $\theta-\theta^{\varepsilon}$ symmetry property of static solutions can be used to construct moving solutions of a spacenoncommutative theory $\widehat{\mathcal{L}}^{\theta}$ from static solutions of the same theory $\widehat{\mathcal{L}}^{\theta}$. Indeed, given $\theta$, if we turn on an electric component such that $\boldsymbol{\varepsilon} \perp \boldsymbol{\theta}$ and $|\boldsymbol{\varepsilon}|<|\boldsymbol{\theta}|$, then with a Lorentz boost we can transform $\theta^{\varepsilon}$ into a space-like $\theta^{\prime}$ proportional to the initial $\theta$. Rescaling $\theta^{\prime} \rightarrow \theta$ we thus obtain a solution (moving with constant velocity) of the space-noncommutative Lagrangian $\widehat{\mathcal{L}}^{\theta}$. I It is this symmetry property that underlies the family of moving solutions found in [38.

We now use SW map and study how the $\theta-\theta^{\varepsilon}$ symmetry acts in the commutative theory. We have to consider the two $\mathrm{SW}$ maps $\mathrm{SW}^{\theta}$ and $\mathrm{SW}^{\theta^{\varepsilon}}$. In general a static solution $\widehat{\phi}, \widehat{A}_{\mu}$ is mapped by $\mathrm{SW}^{\theta}$ and $\mathrm{SW}^{\theta^{\varepsilon}}$ into two different commutative solutions, however if $\widehat{A}_{0}=0$ then $\mathrm{SW}^{\theta}=\mathrm{SW}^{\theta^{\varepsilon}}$. This can be seen from the index structure of SW map. In general we have [cf. paragraph before (34)36]

$$
\begin{aligned}
A_{\mu} & =\widehat{A}_{\mu}+\sum_{n>s} \Theta^{(n)} \partial^{(n+s)} \widehat{A}^{(n-s)} \widehat{A}_{\mu} \\
\phi & =\widehat{\phi}+\sum_{n>s} \Theta^{(n)} \partial^{(n+s)} \widehat{A}^{(n-s)} \widehat{\phi}
\end{aligned}
$$

where the number of times $n, n+s, n-s$ that $\Theta, \partial, \widehat{A}$ appear is dictated by dimensional analysis. In (40) we do not specify which $\partial$ acts on which $\widehat{A}$ and we do not specify the coefficients of each addend. Because of the index structure we notice that $\Theta^{0 i}$ never enters (40) if $\widehat{\phi}, \widehat{A}$ are time independent and $\widehat{A}_{0}=0$. The commutative fields $\phi, A_{i}$ corresponding to $\widehat{\phi}, \widehat{A}_{i}(i \neq 0)$ are solution of both $\mathcal{L}^{\theta}$ and $\mathcal{L}^{\theta^{\varepsilon}}$. Here $\mathcal{L}^{\theta}$ and $\mathcal{L}^{\theta^{\varepsilon}}$ are the commutative Lagrangians corresponding to $\widehat{\mathcal{L}}^{\theta}$

\footnotetext{
${ }^{\mathrm{d}}$ For example, without loss of generality, consider $G=\eta$ and $\theta$ given by $\theta^{12}=-\theta^{21} \neq 0$ with all others $\theta^{\mu \nu}$ vanishing. Let also $\theta^{\varepsilon}$ be given by $\theta^{\varepsilon 12}=\theta^{12}, \theta^{\varepsilon} 02 \neq 0$ all others $\theta^{\varepsilon} \mu \nu$ vanishing. The static solution $\widehat{\phi}(x ; \theta), \widehat{A}_{\mu}(x ; \theta)$ of $\widehat{\mathcal{L}}^{\theta}$ is also a solution of $\widehat{\mathcal{L}}^{\theta^{\varepsilon}}$. Under the Lorentz boost $x^{\mu} \rightarrow x^{\prime \mu}=\Lambda_{\nu}^{\mu} x^{\nu}$ given by $t^{\prime}=\gamma(t+v x), x^{\prime}=\gamma(x+v t), y^{\prime}=y, z^{\prime}=z$ with $v=-\theta^{\varepsilon 02} / \theta^{12}$ the only nonvanishing component of $\theta^{\varepsilon^{\prime}}$ is $\left(\theta^{\varepsilon^{\prime}}\right)^{12}=\theta^{12} / \gamma$. Now notice that for generic $\theta^{\varepsilon}$ and Lorentz transformation $\Lambda$ we have: If $\widehat{\phi}\left(x ; \theta^{\varepsilon}\right)$ is a solution of $\widehat{\mathcal{L}}^{\theta^{\varepsilon}}$ then $\widehat{\phi}^{\prime}\left(x ; \Lambda \theta^{\varepsilon} \Lambda^{T}\right) \equiv \widehat{\phi}\left(\Lambda^{-1} x ; \theta^{\varepsilon}\right)$ is a solution of $\widehat{\mathcal{L}}^{\Lambda \theta^{\varepsilon} \Lambda^{T}}$. Similarly for $\widehat{A}_{\mu}$. In our case we obtain that $\widehat{\phi}^{\prime}(t, x, y, z, \theta / \gamma) \equiv \widehat{\phi}(\gamma(x-$ $v t), y, z, \theta)$ and $\widehat{A}_{\mu}^{\prime}$ solve $\widehat{\mathcal{L}}^{\theta / \gamma}$. Rescaling $\theta \rightarrow \gamma \theta$ we conclude that $\widehat{\phi}(\gamma(x-v t), y, z, \gamma \theta)$ and $\Lambda_{\mu}^{\nu} \widehat{A}_{\nu}(\gamma(x-v t), y, z, \gamma \theta)$ solve $\widehat{\mathcal{L}}^{\theta}$. Here $\widehat{\mathcal{L}}^{\theta}$ is arbitrary, in particular we obtain the moving solitons studied in [38], 41]. For multi-solitons with arbitrary relative motion see [39, 41].
} 
and $\widehat{\mathcal{L}}^{\theta^{\varepsilon}}$ via SW map: $\mathcal{L}^{\theta} \equiv \widehat{\mathcal{L}}\left[\widehat{A}(A, \phi), \widehat{\phi}(A, \phi), G, \star_{\theta}\right]+$ tot.der. (similarly for $\mathcal{L}^{\theta^{\varepsilon}}$ ). The results of this subsection holds also when $\theta$ is $x^{i}$-dependent.

\subsection{Dirac-Born-Infeld Lagrangian}

We now apply this construction to the case of Dirac-Born-Infeld Lagrangian that (for slowly varying fields) is invariant in form under SW map and the change of variables (19). We thus conclude that the static fields $\widehat{\phi}, \widehat{A}_{i}$ solve both the $\mathcal{L}_{\mathrm{DBI}}(F+$ $\left.b, \phi, g, g_{s}\right)$ and the $\mathcal{L}_{\mathrm{DBI}}\left(F+b^{\varepsilon}, \phi, g^{\varepsilon}, g_{s}^{\varepsilon}\right)$ EOM. Actually we are free to impose the invariance of the closed string coupling constant $g_{s}$ under this $\theta-\theta^{\varepsilon}$ transformation. From (19) we see that then the open string coupling constant $G_{s}$ rescales into $G_{s}^{\varepsilon}$ where

$$
G_{s}^{\varepsilon}=G_{s} \sqrt{\frac{\operatorname{det}\left(G^{-1}+\theta^{\varepsilon} / \alpha\right)}{\operatorname{det}\left(G^{-1}+\theta / \alpha\right)}} .
$$

One can be concerned that this $\theta-\theta^{\varepsilon}$ symmetry might hold only in the slowly varying fields approximation. In this subsection we further study this symmetry, we will see that it is an exact symmetry of static configurations of DBI Lagrangian. In order to have a more explicit formulation of the $\theta-\theta^{\varepsilon}$ symmetry we consider (rigid) coordinate transformations $x \rightarrow x^{\prime \prime}$ and $x \rightarrow x^{\prime}$ that respectively orthonormalize $g^{\varepsilon}$ and $g$, while preserving time independence of the transformed $\phi, A$ fields. From now on we set the noncommutative open string metric $G=\eta$. We study the case where the electric and magnetic components of $\theta^{\mu \nu}$ are perpendicular $\boldsymbol{\varepsilon} \perp \boldsymbol{\theta}$. (We could have chosen a more general case, the final result (54) is independent from the $\boldsymbol{\varepsilon} \perp \boldsymbol{\theta}$ choice). With $\boldsymbol{\varepsilon} \perp \boldsymbol{\theta}$, without loss of generality we consider

$$
\theta \equiv-\theta^{\varepsilon 12}=\theta^{\varepsilon 21} \quad, \quad \varepsilon \equiv \theta^{\varepsilon 02}=-\theta^{\varepsilon 20} \quad \text { all others } \theta^{\varepsilon \mu \nu}=0 \text {. }
$$

Then

$$
\begin{gathered}
g_{00}^{\varepsilon}=-1-\frac{\varepsilon^{2}}{\alpha^{\prime 2}+\theta^{2}-\varepsilon^{2}}, \quad g_{01}^{\varepsilon}=\frac{-\varepsilon \theta}{\alpha^{\prime 2}+\theta^{2}-\varepsilon^{2}}, \quad g^{\varepsilon}{ }_{11}=1-\frac{\theta^{2}}{\alpha^{\prime 2}+\theta^{2}-\varepsilon^{2}} \\
g^{\varepsilon}{ }_{22}=\frac{\alpha^{\prime 2}}{\alpha^{\prime 2}+\theta^{2}-\varepsilon^{2}}, \quad g_{33}^{\varepsilon}=1 ; \quad b_{02}^{\varepsilon}=\frac{\varepsilon}{\alpha^{\prime 2}+\theta^{2}-\varepsilon^{2}}, \quad b^{\varepsilon}{ }_{12}=\frac{\theta}{\alpha^{\prime 2}+\theta^{2}-\varepsilon^{2}}
\end{gathered}
$$

all other $g^{\varepsilon}{ }_{\mu \nu}, b^{\varepsilon}{ }_{\mu \nu}$ components vanishing. Under

$$
x \rightarrow x^{\prime \prime}=\left(\Gamma^{\varepsilon}\right)^{-1} x ; \quad \Gamma^{\varepsilon} \equiv\left(\begin{array}{cccc}
\sqrt{1-\frac{\varepsilon^{2}}{\alpha^{\prime 2}+\theta^{2}}} & -\frac{\varepsilon \theta}{\alpha^{\prime} \sqrt{\alpha^{\prime 2}+\theta^{2}}} & 0 & 0 \\
0 & \sqrt{1+\frac{\theta^{2}}{\alpha^{\prime 2}}} & 0 & 0 \\
0 & 0 & \sqrt{1+\frac{\theta^{2}-\varepsilon^{2}}{\alpha^{\prime 2}}} & 0 \\
0 & 0 & 0 & 1
\end{array}\right)
$$


[obtained composing (20) with a Lorentz transformation] we have

$$
\begin{gathered}
g^{\varepsilon} \rightarrow \eta, \quad b^{\varepsilon} \rightarrow b^{\prime \prime}=\Gamma^{\varepsilon T} b^{\varepsilon} \Gamma^{\varepsilon} \\
\phi(x) \rightarrow \phi^{\prime \prime}\left(x^{\prime \prime}\right)=\phi\left(\Gamma^{\varepsilon} x^{\prime \prime}\right), \quad A(x) \rightarrow A^{\prime \prime}\left(x^{\prime \prime}\right)=\Gamma^{\varepsilon T} A\left(\Gamma^{\varepsilon} x^{\prime \prime}\right)
\end{gathered}
$$

In (46) $\phi^{\prime \prime}, A^{\prime \prime}$ are $x^{\prime \prime 0}$ independent and $A_{0}^{\prime \prime}=0$ if $\phi, A$ are $x^{0}$ independent and $A_{0}=0$. The nonzero components of $b^{\prime \prime}$ are

$$
e^{\prime \prime} \equiv-b^{\prime \prime}{ }_{02}=\frac{-\varepsilon}{\alpha^{\prime} \sqrt{\alpha^{\prime 2}+\theta^{2}}} \quad, \quad b^{\prime \prime} \equiv b_{12}^{\prime \prime}=\frac{\theta}{\alpha^{\prime 2}} \sqrt{1-\frac{\varepsilon^{2}}{\alpha^{\prime 2}+\theta^{2}}}
$$

Obviously the fields $\phi^{\prime \prime}, A^{\prime \prime}$ satisfy the $\mathcal{L}_{\text {DBI }}\left(F^{\prime \prime}+b^{\prime \prime}, \phi^{\prime \prime}, \eta, g_{s}\right)$ EOM if $\phi, A$ satisfy the $\mathcal{L}_{\mathrm{DBI}}\left(F+b^{\varepsilon}, \phi, g^{\varepsilon}, g_{s}\right)$ EOM.

In order to obtain the $x \rightarrow x^{\prime}$ transformation we consider $\varepsilon=0$ in (43)-(47), indeed

$$
\theta=\left.\theta^{\varepsilon}\right|_{\varepsilon=0} \quad, \quad g=\left.g^{\varepsilon}\right|_{\varepsilon=0} \quad, \quad b=\left.b^{\varepsilon}\right|_{\varepsilon=0}
$$

and under

$$
x \rightarrow x^{\prime}=\Gamma^{-1} x \quad,\left.\quad \Gamma \equiv \Gamma^{\varepsilon}\right|_{\varepsilon=0}
$$

we have $g \rightarrow \eta, b \rightarrow b^{\prime}=\Gamma^{T} b \Gamma$. The transformed solution $\phi^{\prime}, A^{\prime}$ satisfies the EOM of $\mathcal{L}_{\mathrm{DBI}}\left(F^{\prime}+b^{\prime}, \phi^{\prime}, \eta, g_{s}\right)$. The nonzero component of $b_{\mu \nu}^{\prime}$ is : $b^{\prime} \equiv b_{12}^{\prime}=\theta / \alpha^{\prime 2}$.

We now compose the $\Gamma^{-1}$ and $\Gamma^{\varepsilon}$ transformations and conclude that given a static solution $\phi^{\prime}\left(x^{\prime}\right), A^{\prime}\left(x^{\prime}\right)$ of $\mathcal{L}_{\mathrm{DBI}}\left(F^{\prime}+b^{\prime}, \phi^{\prime}, \eta, g_{s}\right)$ with $A_{0}^{\prime}=0$ and $b_{12}^{\prime}=b^{\prime}$ (all others $b_{\mu \nu}^{\prime}$ vanishing) then $\phi^{\prime \prime}\left(x^{\prime \prime}\right), A^{\prime \prime}\left(x^{\prime \prime}\right)$ is a static solution of $\mathcal{L}_{\mathrm{DBI}}\left(F^{\prime \prime}+\right.$ $\left.b^{\prime \prime}, \phi^{\prime \prime}, \eta, g_{s}\right)$ where $A_{0}^{\prime \prime}=0$ and

$$
\begin{gathered}
\Upsilon \equiv \Gamma^{-1} \Gamma^{\varepsilon}, \quad x^{\prime \prime}=\Upsilon^{-1} x^{\prime}, \\
\phi^{\prime \prime}\left(x^{\prime \prime}\right)=\phi^{\prime}\left(\Upsilon x^{\prime \prime}\right), \quad A^{\prime \prime}\left(x^{\prime \prime}\right)=\Upsilon^{T} A^{\prime}\left(\Upsilon x^{\prime \prime}\right),
\end{gathered}
$$

the nonzero components of $b_{\mu \nu}^{\prime \prime}$, expressed in terms of $e^{\prime \prime}$ and of $b^{\prime}$ are

$$
e^{\prime \prime} \equiv-b^{\prime \prime}{ }_{02} \quad, \quad b^{\prime \prime} \equiv b^{\prime \prime}{ }_{12}=\sqrt{1-\alpha^{2} e^{\prime \prime 2}} b^{\prime} .
$$

The explicit expression of $\Upsilon$ is

$$
\Upsilon=\left(\begin{array}{cccc}
\sqrt{1-\alpha^{\prime 2} e^{\prime \prime 2}} & \alpha^{\prime 2} e^{\prime \prime} b^{\prime} & 0 & 0 \\
0 & 1 & 0 & 0 \\
0 & 0 & \sqrt{1-\alpha^{\prime 2} e^{\prime \prime 2}} & 0 \\
0 & 0 & 0 & 1
\end{array}\right)
$$


Let $\mathcal{A}^{\prime}$ be the gauge potential of $\mathcal{F}^{\prime}=F^{\prime}+b^{\prime}$, the $\phi^{\prime}, A^{\prime}$ and $b^{\prime}$ transformations then read

$$
\phi^{\prime \prime}\left(x^{\prime \prime}\right)=\phi^{\prime}\left(\Upsilon x^{\prime \prime}\right), \quad \mathcal{A}_{i}^{\prime \prime}\left(x^{\prime \prime}\right)=\Upsilon_{i}^{j} \mathcal{A}_{j}^{\prime}\left(\Upsilon x^{\prime \prime}\right), \quad \mathcal{A}_{0}^{\prime \prime}\left(x^{\prime \prime}\right)=e^{\prime \prime} x_{2}^{\prime \prime}
$$

If $\phi^{\prime}, \mathcal{A}_{i}^{\prime}$ is a static solution of $\mathcal{L}_{\mathrm{DBI}}$ then $\phi^{\prime \prime}, \mathcal{A}_{\mu}^{\prime \prime}$ is a new static solution. Notice now that in this section we have never used that the $F_{i j}^{\prime}$ that appear in $\mathcal{F}^{\prime}=F^{\prime}+b^{\prime}$ vanish at spatial infinity; therefore (54) with $\Upsilon^{j}{ }_{i}$ as in (53) holds for arbitrary static fields configurations $\phi^{\prime}, \mathcal{A}^{\prime}$ with $\mathcal{A}_{0}^{\prime}=0$.

For future reference we summarise the relation between the coordinate systems $x, x^{\prime}, x^{\prime \prime}$ in the following commutative diagram

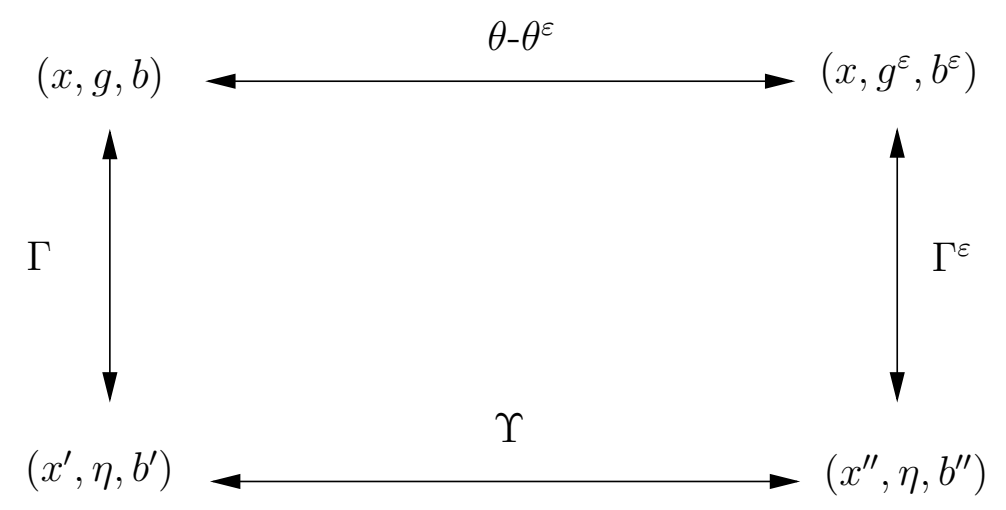

The quantities on the left hand side can be obtained from those on the right hand side simply by setting $\varepsilon=0$.

\subsection{Symmetries in extended target space}

Using the $\theta-\theta^{\varepsilon}$ symmetry property of noncommutative Lagrangians we have constructed new solutions (54) of $\mathcal{L}_{\mathrm{DBI}}$; the field strength in these solutions has a constant term $b_{\mu \nu}^{\prime \prime}$ with both electric and magnetic components. Here we illustrate the commutative $\mathcal{L}_{\mathrm{DBI}}$ symmetry underlying this family of solutions. As a consequence we see that the $\theta-\theta^{\varepsilon}$ symmetry of $\mathcal{L}_{\mathrm{DBI}}$ is exact and not restricted to the slowly varying fields approximation. We split $\mathcal{F}^{\prime}$ in its electric and magnetic components $\mathcal{E}^{\prime}, \mathcal{B}^{\prime}$. We then consider the Legendre transformation of $\mathcal{L}_{\mathrm{DBI}}$ with $C=0$ [cf. (10)]

$$
\check{H}\left(\mathcal{E}^{\prime}, \mathcal{H}^{\prime}, \phi^{\prime}\right)=\frac{1}{g_{s}} \mathcal{B}^{\prime} \cdot \mathcal{H}^{\prime}+\mathcal{L}_{\mathrm{DBI}} \quad, \quad \text { where } \quad \mathcal{H}_{i}^{\prime}=-g_{s} \frac{\partial \mathcal{L}_{\mathrm{DBI}}}{\partial \mathcal{B}^{\prime i}}
$$


For time independent fields $\phi^{\prime}, \mathcal{A}_{\mu}^{\prime}$ we have $\left[\alpha^{\prime}=1\right.$ in (57) and (58)]

$$
\begin{aligned}
& \mathcal{L}=\frac{-1}{g_{s}}\left[1+\left(\nabla^{\prime} \phi^{\prime}\right)^{2}+\mathcal{B}^{\prime 2}-\mathcal{E}^{\prime 2}+\left(\nabla^{\prime} \phi^{\prime} \cdot \mathcal{B}^{\prime}\right)^{2}+\left(\nabla^{\prime} \phi^{\prime} \cdot \mathcal{E}^{\prime}\right)^{2}-\left(\nabla^{\prime} \phi^{\prime}\right)^{2} \mathcal{E}^{\prime 2}-\left(\mathcal{B}^{\prime} \cdot \mathcal{E}^{\prime}\right)^{2}\right]^{\frac{1}{2}} \\
& \mathcal{H}_{i}^{\prime}=\frac{\mathcal{B}_{i}^{\prime}+\left(\mathcal{B}^{\prime} \cdot \nabla^{\prime} \phi^{\prime}\right) \partial_{i}^{\prime} \phi^{\prime}-\left(\mathcal{B}^{\prime} \cdot \mathcal{E}^{\prime}\right) \mathcal{E}_{i}^{\prime}}{\left[1+\left(\nabla^{\prime} \phi^{\prime}\right)^{2}+\mathcal{B}^{\prime 2}-\mathcal{E}^{\prime 2}+\left(\nabla^{\prime} \phi^{\prime} \cdot \mathcal{B}^{\prime}\right)^{2}+\left(\nabla^{\prime} \phi^{\prime} \cdot \mathcal{E}^{\prime}\right)^{2}-\left(\nabla^{\prime} \phi^{\prime}\right)^{2} \mathcal{E}^{\prime 2}-\left(\mathcal{B}^{\prime} \cdot \mathcal{E}^{\prime}\right)^{2}\right]^{\frac{1}{2}}}
\end{aligned}
$$

We also have that the EOM (乐) imply $\mathcal{H}^{\prime}=-\nabla^{\prime} \chi^{\prime}$ and $\mathcal{E}^{\prime}=-\nabla^{\prime} \psi^{\prime},\left(\psi^{\prime}=-\mathcal{A}_{0}^{\prime}\right)$. As shown in [10] it follows that $\check{H}\left(\psi^{\prime}, \chi^{\prime}, \phi^{\prime}\right)$ is the action of a space-like 3-brane immersed in a target space of coordinates $X^{\prime A}=\left\{\alpha^{\prime} \psi^{\prime}, \alpha^{\prime} \chi^{\prime}, \alpha^{\prime} \phi^{\prime}, x^{\prime}\right\}$ and metric $\eta=\operatorname{diag}(-1,-1,1,1,1,1)$

$$
\Sigma_{\check{H}} \equiv \int d^{3} x^{\prime} \check{H}=\frac{-1}{g_{s} \alpha^{\prime 2}} \int d^{3} x^{\prime} \sqrt{\operatorname{det}\left(\eta_{A B} \frac{\partial X^{\prime A}}{\partial x^{\prime i}} \frac{\partial X^{\prime B}}{\partial x^{\prime j}}\right)} .
$$

It is the $S O(2,4)$ symmetry 10 of this static gauge action that is relevant in our context: Consider the Lorentz transformation $Y^{A}=\Lambda_{B}^{A} X^{\prime B}$ (where $X^{\prime i}=x^{\prime i}$ ) and express $Y^{A}$ as $Y^{A}=Y^{A}\left(y^{i}\right)$ (where $\left.Y^{i}=y^{i}\right)$ so that we are still in static gauge; the action (59) is invariant under $X^{\prime A}\left(x^{\prime i}\right) \rightarrow Y^{A}\left(y^{i}\right)$. In particular consider a boost in the $\alpha^{\prime} \psi^{\prime}, x_{2}^{\prime}$ plane with velocity $\beta=-\alpha^{\prime} e^{\prime \prime}$. Under this boost

$$
\begin{gathered}
\left(\begin{array}{c}
\alpha^{\prime} \psi^{\prime} \\
x_{2}^{\prime}
\end{array}\right) \longrightarrow\left(\begin{array}{c}
\alpha^{\prime} \psi^{\prime \prime} \\
x_{2}^{\prime \prime}
\end{array}\right)=\left(\begin{array}{cc}
\gamma & \gamma \beta \\
\gamma \beta & \gamma
\end{array}\right)\left(\begin{array}{c}
\alpha^{\prime} \psi^{\prime} \\
x_{2}^{\prime}
\end{array}\right) \\
x_{1}^{\prime \prime}=x_{1}^{\prime}, \quad x_{3}^{\prime \prime}=x_{3}^{\prime} \quad, \quad \chi^{\prime \prime}\left(x^{\prime \prime}\right)=\chi^{\prime}\left(x^{\prime}\right), \quad \phi^{\prime \prime}\left(x^{\prime \prime}\right)=\phi^{\prime}\left(x^{\prime}\right)
\end{gathered}
$$

where $\gamma^{-1}=\sqrt{1-\beta^{2}}=\sqrt{1-\alpha^{\prime 2} e^{\prime \prime 2}}$. If $\psi^{\prime}=0$ we have

$$
\begin{array}{ccc}
x^{i}=\Upsilon^{i}{ }_{j} x^{\prime \prime} j & , \quad \psi^{\prime \prime}=-e^{\prime \prime} x_{2}^{\prime \prime} & \\
\mathcal{H}_{2}^{\prime \prime}\left(x^{\prime \prime}\right)=\gamma^{-1} \mathcal{H}_{2}^{\prime}\left(x^{\prime}\right), & \mathcal{H}_{q}^{\prime \prime}\left(x^{\prime \prime}\right)=\mathcal{H}_{q}^{\prime}\left(x^{\prime}\right) & q=1,3 \\
\partial_{2}^{\prime \prime} \phi^{\prime \prime}\left(x^{\prime \prime}\right)=\gamma^{-1} \partial_{2}^{\prime} \phi^{\prime}\left(x^{\prime}\right), & \partial_{q}^{\prime \prime} \phi^{\prime \prime}\left(x^{\prime \prime}\right)=\partial_{q}^{\prime} \phi^{\prime}\left(x^{\prime}\right) & q=1,3 .
\end{array}
$$

We want to show that the magnetic induction

$$
\mathcal{B}^{\prime \prime}{ }^{i}=g_{s} \frac{\partial \check{H}\left(\mathcal{E}^{\prime \prime}, \mathcal{H}^{\prime \prime}, \phi^{\prime \prime}\right)}{\partial \mathcal{H}_{i}^{\prime \prime}}
$$

is the same as that obtained from (54), i.e.

$$
\mathcal{B}_{2}^{\prime \prime}\left(x^{\prime \prime}\right)=\mathcal{B}_{2}^{\prime}\left(x^{\prime}\right), \quad \mathcal{B}_{q}^{\prime \prime}\left(x^{\prime \prime}\right)=\gamma^{-1} \mathcal{B}_{q}^{\prime}\left(x^{\prime}\right) \quad q=1,3
$$

We can equivalently show that (63) and (65) imply (62). This is done using the explict expression (58). 
We end this subsection observing that the $S O(2)$ subgroup of $S O(2,4)$ that acts on $\psi^{\prime}, \chi^{\prime}$ is simply the $S L(2, R)$ subgroup of duality rotations (11) that leaves invariant $g_{s}$ and $C=0$.

\subsection{BPS solutions for (NC)DBI with a scalar field}

In order to study BPS solutions [2] we minimize the energy functional (Hamiltonian) relative to NCDBI theory. The Hamiltonian formalism in space-time noncommutative theories is studied in [37]. For theories (like NCDBI) invariant under time translations the Hamiltonian defined in [37] is the generator of this symmetry. Here we are interested in the static case with $\widehat{A}_{0}=0$, then $\hat{D}_{0} \widehat{\phi}=0, \hat{E}_{i}=-\widehat{F}_{0 i}=0$ and the Hamiltonian becomes as usual just minus the integral over space of the Lagrangian density

$$
\begin{aligned}
\hat{\Sigma}_{\mathrm{DBI}} & =\int d^{3} x-\widehat{\mathcal{L}}_{\mathrm{DBI}}=\int d^{3} x \frac{1}{\alpha^{\prime 2} G_{s}} \sqrt{\operatorname{det}\left(\eta_{i j}+\alpha^{\prime} \widehat{F}_{i j}+\alpha^{\prime 2} \hat{D}_{i} \widehat{\phi} \hat{D}_{j} \widehat{\phi}\right)}+O(\partial \widehat{F}, \partial \hat{D} \widehat{\phi}) \\
& =\int d^{3} x \frac{1}{\alpha^{\prime 2} G_{s}} \sqrt{1+\alpha^{\prime 2}(\hat{\boldsymbol{D}} \widehat{\phi})^{2}+\alpha^{\prime 2} \hat{\boldsymbol{B}}^{2}+\alpha^{\prime 4}(\hat{\boldsymbol{B}} \cdot \hat{\boldsymbol{D}} \widehat{\phi})^{2}}+O(\partial \widehat{F}, \partial \hat{D} \widehat{\phi})
\end{aligned}
$$

Notice that $-\alpha^{\prime 2} G_{s} \widehat{\mathcal{L}} \geq 1+\alpha^{\prime 2}|\hat{\boldsymbol{B}} \cdot \hat{\boldsymbol{D}} \widehat{\phi}|$, so that

$$
\hat{\Sigma}_{\mathrm{DBI}} \geq\left|\hat{Z}_{m}\right|+\int d^{3} x \frac{1}{\alpha^{\prime 2} G_{s}}
$$

where $\hat{Z}_{m}$ is the topological charge given by

$$
\hat{Z}_{m}=\frac{1}{\alpha^{\prime 2} G_{s}} \int d^{3} x \hat{\boldsymbol{B}} \cdot \hat{\boldsymbol{D}} \widehat{\phi}=\frac{1}{\alpha^{\prime 2} G_{s}} \int d^{3} x \hat{\boldsymbol{D}} \cdot(\hat{\boldsymbol{B}} \widehat{\phi})
$$

in the last equality we have used the Bianchi identity $\hat{\boldsymbol{D}} \cdot \hat{\boldsymbol{B}}=0$. We rewrite the argument of the square root as $\left(1 \pm \alpha^{\prime 2} \hat{\boldsymbol{B}} \cdot \hat{\boldsymbol{D}} \widehat{\phi}\right)^{2}+\alpha^{\prime 2}(\hat{\boldsymbol{B}} \mp \hat{\boldsymbol{D}} \widehat{\phi})^{2}$, for fixed $\hat{Z}_{m}$ we see that $\hat{\Sigma}_{\mathrm{DBI}}$ is minimal if the BPS equation

$$
\hat{B}_{i}= \pm \hat{D}_{i} \widehat{\phi}
$$

is satisfied (with plus sign if $\hat{Z}_{m}>0$, minus sign otherwise). The energy of this configuration, apart from the addend $\left(\alpha^{\prime 2} G_{s}\right)^{-1}$ (D3-brane tension), equals the absolute value of the topological charge.

Equations (69) are also the BPS equations for noncommutative electromagnetism whose energy functional is

$$
\begin{aligned}
\hat{\Sigma}_{\mathrm{EM}} & =\frac{1}{2 G_{s}} \int d^{3} x \hat{\boldsymbol{B}} \star \hat{\boldsymbol{B}}+\hat{\boldsymbol{D}} \widehat{\phi} \star \hat{\boldsymbol{D}} \widehat{\phi} \\
& =\frac{1}{2 G_{s}} \int d^{3} x\left(\hat{D}_{i} \widehat{\phi} \pm \hat{B}_{i}\right) \star\left(\hat{D}^{i} \widehat{\phi} \pm \hat{B}^{i}\right) \mp \partial_{i}\left(\hat{B}^{i} \star \widehat{\phi}+\widehat{\phi} \star \hat{B}^{i}\right)
\end{aligned}
$$


The BPS equations for the commutative DBI Lagrangian $\mathcal{L}_{\text {DBI }}\left(\mathcal{F}, \phi, g, g_{s}\right)$ are 9 , 10, 11] $\mathcal{E}_{i}=-\mathcal{F}_{0 i}=0$ and

$$
\mathcal{B}_{i}= \pm \partial_{i} \phi
$$

they can be obtained as in the noncommutative case. Here we have considered a metric $g_{\mu \nu}$ with block diagonal form $g_{00}=-1, g_{0 i}=0$, so that $\mathcal{B}_{i}=g_{i j} \mathcal{B}^{j}$ with $\mathcal{B}^{i}=\mathcal{F}^{* 0 i}=\frac{1}{2} \sqrt{g^{-1}} \epsilon^{i j k} \mathcal{F}_{i j}$. The Bianchi identity $\partial_{i} B^{i}=0$ implies $\partial^{i} \partial_{i} \phi=0$; DBI monopoles corresponds to isolated singularities of the harmonic function $\phi$. Using the definition of $\mathcal{H}$ [cf. (56) and (58)] we see that (71) is equivalent to

$$
\mathcal{H}_{i}= \pm \partial_{i} \phi \quad, \quad \partial^{i} \partial_{i} \phi=0
$$

The energy of a BPS state equals the charge $Z_{m}=\frac{1}{\alpha^{\prime 2} g_{s}} \int \mathcal{B}^{i} \partial_{i} \phi=\frac{ \pm 1}{\alpha^{\prime 2} g_{s}} \int \mathcal{B}^{i} \mathcal{H}_{i}$.

A solution to (71) in a reference system with metric $g$ and background $b$ as in (55) is

$$
\phi=\frac{-\theta}{\alpha^{\prime 2}} x^{3}-\frac{1}{2 r} \quad, \quad \mathcal{B}_{i}=-\partial_{i} \phi \quad ; \quad r^{2}=g_{i j} x^{i} x^{j}=\left(x^{3}\right)^{2}+\frac{\left(x^{1}\right)^{2}+\left(x^{2}\right)^{2}}{1+\theta^{2} / \alpha^{\prime 2}}
$$

where $\frac{1}{2 r}=\frac{q_{m}}{4 \pi r}$ with $q_{m}$ the magneton charge $q_{m}=2 \pi$. This solution describes a D1-string ending on a D3-brane. Because of the magnetic backgound field $b$ on the brane the string is not perpendicular to the brane, in (73) the string is vertical and the brane is tilted, see Fig. 2 (with axes $\bar{x}^{3}$ and $\alpha^{\prime} \bar{\phi}$ renamed $x^{3}$ and $\alpha^{\prime} \phi$ ). We call $\pi / 2-\zeta$ the angle between the string and the brane, $\tan \zeta=\theta / \alpha^{\prime}$. The magnetic force acting on the end of the D1-string is compensated by the tension of the D1-string [16.

A solution of (69) with space-like noncommutativity given by $\theta^{12}=-\theta$, (all others $\theta^{\mu \nu}=0$ ) has been studied in 29. It is a static solution with $\widehat{A}_{0}=\widehat{A}_{3}=0$. Far away from the origin the $\hat{B}$ field is the sum of two terms. The first term is that of a point-like negative charged magneton placed at the origin, the second term vanishes for $x_{3}<<1$ while for $x_{3}>>1$ we have

$$
\hat{B}_{3}=\frac{2}{\theta} e^{-\frac{\left(x^{1}\right)^{2}+\left(x^{2}\right)^{2}}{\theta}}, \quad \widehat{\phi}=\frac{-2 x^{3}}{\theta} e^{-\frac{\left(x^{1}\right)^{2}+\left(x^{2}\right)^{2}}{\theta}}
$$

The energy in the interval $\left|x^{3}\right| \leq L$ with $L>>1$ grows linearly with $L$ and is given by $\hat{\Sigma}=\frac{2 \pi L}{G_{s} \theta}$. As we see from (74) this energy is concentrated around the positive $x^{3}$ axis. We can think of a semi-infinite string along the positive $x^{3}$ axis with a tension

$$
\hat{T}=\frac{2 \pi}{G_{s} \theta}
$$

attached to it there is a magnetic monopole smeared around the origin. This configuration is shown to describe the D1-string ending on the D3-brane (73). A first 
evidence is the correspondence between the tension of the noncommutative string and that of the D1-string [29], then in [30 the spectrum of small fluctuations around (a limit of) this solution is studied and found in agreement with the expectations from string theory. This suggests that the noncommutative BPS equations (69) corresponds, via SW map, to the commutative BPS ones (71). More precisely [17, 33, 34], for space noncommutativity the noncommutative BPS equations (69) are mapped into the nonlinear BPS equations of DBI theory. These nonlinear BPS equations are in turn equivalent to the linear BPS equation (71) via a target space rotation in the $\alpha^{\prime} \phi, x_{3}$ plane. If the magnetic part of $\theta$ is given by $\theta^{12}=-\theta$ as in (73) the $\alpha^{\prime} \phi, x_{3}$ rotation is by an angle $\zeta, \tan \zeta=\theta / \alpha^{\prime}$

$$
\left(\begin{array}{c}
\alpha^{\prime} \phi \\
x^{3}
\end{array}\right) \longrightarrow\left(\begin{array}{c}
\alpha^{\prime} \bar{\phi} \\
\bar{x}^{3}
\end{array}\right)=\left(\begin{array}{cc}
\cos \zeta & -\sin \zeta \\
\sin \zeta & \cos \zeta
\end{array}\right)\left(\begin{array}{c}
\alpha^{\prime} \phi \\
x^{3}
\end{array}\right)
$$

we have 33 .

$$
\widehat{\phi}\left(x^{1}, x^{2}, x^{3}\right) \stackrel{\mathrm{SW} \operatorname{map}}{\longleftrightarrow} \phi\left(x^{1}, x^{2}, x^{3}\right) \stackrel{\zeta \text {-rot. }}{\longleftrightarrow} \bar{\phi}\left(x^{1}, x^{2}, \bar{x}^{3}\right)
$$

where $\phi\left(x^{1}, x^{2}, x^{3}\right)$ satisfies the nonlinear BPS equation and $\bar{\phi}\left(x^{1}, x^{2}, \bar{x}^{3}\right)$ the linear one. The profiles of $\phi$ and $\bar{\phi}$ are described in the following figures

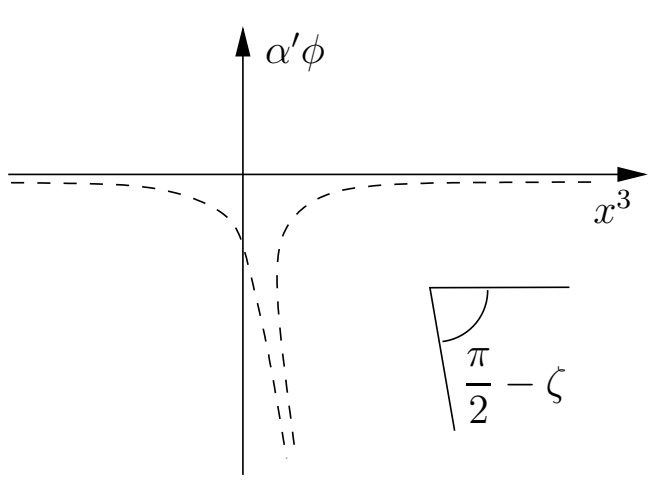

Fig. 1

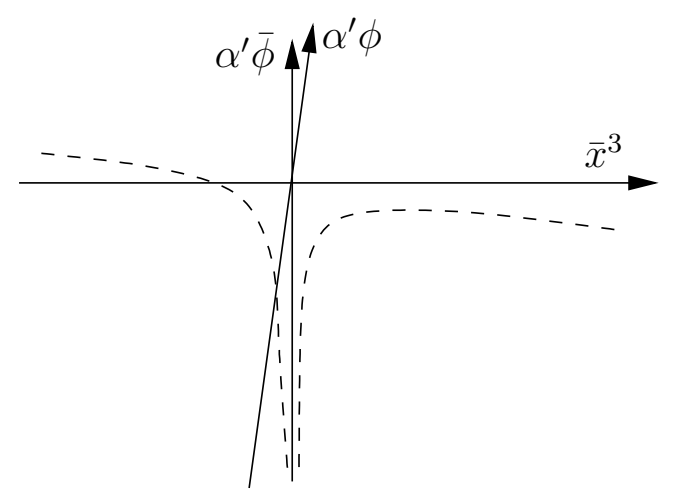

Fig. 2

As shown in 35, the $S O(2)$ rotation group (76) is a symmetry of DBI action. For static configurations the $S O(2)$ rotation group (76) is a subgroup of the $S O(2,4)$ symmetry group discussed after formula (58). Actually the $\zeta$-rotation (76) commutes with the $\Gamma, \Upsilon$ and $\Gamma^{\varepsilon}$ transformations, so that [recall diagram (55)] it commutes with the $\theta-\theta^{\varepsilon}$ transformation: the $\zeta$ angle is independent from the noncommutativity parameter $\varepsilon$. Since the rotation (76) is a symmetry we have that

${ }^{\mathrm{e}}$ In [33] the closed string variables are $\left\{g^{M}=\eta, b^{M}\right\}$ so that the open ones are $\left\{G^{M} \neq \eta, \theta^{M}\right\}$. On the other hand we have chosen $\{G=\eta, \theta\}$ so that $\{g \neq \eta, b\}$. The rotation angle $\zeta$ is the same $\tan \zeta=\theta / \alpha^{\prime}=\alpha^{\prime} b^{M}$ in both descriptions because the coordinate transformation that maps $\left\{g^{M}, b^{M}\right\} \rightarrow\{g, b\}$ commutes with the $\zeta$-rotation. 
the energy of a field configuration is left invariant under (76). It is instructive to see this explicitly for static field configurations, some of the techniques in (78)-(87) will be used later. We also show that the magnetic and electric charges are independent from the $\zeta$-rotation. Under the $\zeta$-rotation $(76)$ we have

$$
\bar{x}^{1}=x^{1}, \quad \bar{x}^{2}=x^{2}, \quad \bar{\chi}(\bar{x})=\chi(x), \quad \bar{\psi}(\bar{x})=\psi(x), \quad \bar{\partial}_{i}=\frac{\partial x^{j}}{\partial \bar{x}^{i}} \partial_{j} \equiv J_{i}{ }^{j} \partial_{j}
$$

and recalling that $\mathcal{H}_{i}=-\partial_{i} \chi$ and $\mathcal{E}_{i}=-\mathcal{F}_{0 i}=-\partial_{i} \psi$,

$$
\overline{\mathcal{H}}_{i}(\bar{x})=J_{i}{ }^{j} \mathcal{H}_{j}(x) \quad, \quad \overline{\mathcal{E}}_{i}(\bar{x})=J_{i}{ }^{j} \mathcal{E}_{j}(x) .
$$

In terms of $\mathcal{E}$ and $\mathcal{H}$ the magnetic induction $\mathcal{B}^{i}=\mathcal{F}^{* 0 i}$ is given by [cf. (56)], $\mathcal{B}^{i}(x)=g_{s} \frac{\delta \Sigma_{\breve{H}}}{\delta \mathcal{H}_{i}(x)}$. The relation between $\mathcal{B}(x)$ and the $\zeta$-rotated field $\overline{\mathcal{B}}(\bar{x})$ is

$$
\mathcal{B}^{i}(x)=g_{s} \frac{\delta \Sigma_{\check{H}}}{\delta \mathcal{H}_{i}(x)}=g_{s} \int d^{3} \bar{y} \frac{\delta \Sigma_{\check{H}}}{\delta \overline{\mathcal{H}}_{j}(\bar{y})} \frac{\delta \overline{\mathcal{H}}_{j}(\bar{y})}{\delta \mathcal{H}_{i}(x)}=\overline{\mathcal{B}}^{j}(\bar{x}) J_{j}{ }^{i} \operatorname{det} J^{-1}
$$

where in the second passage we have used that $\frac{\delta \overline{\mathcal{E}}}{\delta \mathcal{H}}=\frac{\delta \bar{\phi}}{\delta \mathcal{H}}=0$ because $J$ does not depend on $\mathcal{H}$. We then have

$$
\partial_{i} \mathcal{B}^{i}(x) d^{3} x=\partial_{i} \overline{\mathcal{B}}^{j}(\bar{x}) J_{j}{ }^{i} d^{3} \bar{x}+\overline{\mathcal{B}}^{j}(\bar{x}) \partial_{i}\left(J_{j}{ }^{i} \operatorname{det} J^{-1}\right) d^{3} x=\bar{\partial}_{i} \overline{\mathcal{B}}^{i}(\bar{x}) d^{3} \bar{x}
$$

in the last passage we used $\partial_{i} \operatorname{det} J^{-1}=\partial_{i}\left(J_{k}^{-1 \ell}\right) \frac{\partial \operatorname{det} J^{-1}}{\partial J^{-1} k_{k}}=\partial_{k}\left(J_{i}^{-1 \ell}\right) J_{\ell}^{k} \operatorname{det} J^{-1}$. The same analysis holds if in (80), (81) we replace $\mathcal{H}$ with $\mathcal{E}$ and thus see that also the electric charge is independent from the $\zeta$-rotation

$$
\frac{1}{g_{s}} \partial_{i} \mathcal{D}^{i}(x) d^{3} x=\frac{1}{g_{s}} \bar{\partial}_{i} \overline{\mathcal{D}}^{i}(\bar{x}) d^{3} \bar{x}
$$

here

$$
\mathcal{D}^{i}=g_{s} \frac{\partial \mathcal{L}_{\mathrm{DBI}}}{\partial \mathcal{E}_{i}}=g_{s} \frac{\partial \check{H}}{\partial \mathcal{E}_{i}}
$$

Similarly we have

$$
\begin{aligned}
\mathcal{B}^{i} \mathcal{H}_{i} d^{3} x & =\overline{\mathcal{B}}^{i} \overline{\mathcal{H}}_{i} d^{3} \bar{x} \\
\mathcal{E}_{i} \mathcal{D}^{i} d^{3} x & =\overline{\mathcal{E}}_{i} \overline{\mathcal{D}}^{i} d^{3} \bar{x}
\end{aligned}
$$

Recalling (56) and the invariance of (59) under the $\zeta$-rotation (76) we obtain that $\mathcal{L}_{\text {DBI }} d^{3} x$ is invariant. The invariance of the energy functional follows from

$$
H(x) d^{3} x=\bar{H}(\bar{x}) d^{3} \bar{x}
$$

where the energy density $H$ is given by the Legendre transformation

$$
H=\frac{1}{g_{s}} \mathcal{E}_{i} \mathcal{D}^{i}-\mathcal{L}_{\mathrm{DBI}}
$$


In (87) $H$ equals the gravitational energy density $-T_{0}^{0}$, with $T_{\mu \nu}=\frac{-2}{\sqrt{g}} \frac{\partial \mathcal{L}_{\mathrm{DBI}}}{\partial g^{\mu \nu}}$ [1]. From (86) one can also show that the string tension is independent from the $\zeta$ rotation.

Since the $\zeta$-rotation (76) is independent from the noncommutativity parameter $\varepsilon$, and since it does not modify the energy, the electric and magnetic charges $\frac{1}{g_{s}} \int d^{3} x \partial_{i} \mathcal{D}^{i}, \int d^{3} x \sqrt{g} \partial_{i} \mathcal{B}^{i}$ and the string tension of a given field configuration, in the following we call SW map what is actually SW map plus $\zeta$-rotation; we also write $\phi, x^{3}$ instead of $\bar{\phi}, \bar{x}^{3}$.

\subsection{BPS type solutions with both electric and magnetic backgrounds}

From Subsection 3.1 and (41) we have that Gross-Nekrasov soliton is also a BPS solution of space-time noncommutative DBI theory. The string tension is now

$$
\hat{T}^{\varepsilon}=\frac{2 \pi}{G_{s}^{\varepsilon} \theta}
$$

Applying SW map to this noncommutative BPS solution we obtain that (73) with $\mathcal{E}_{2}=-b^{\varepsilon}{ }_{02}, \mathcal{E}_{1}=\mathcal{E}_{2}=0$ solves $\mathcal{L}_{\mathrm{DBI}}\left(F+b^{\varepsilon}, \phi, g^{\varepsilon}, g_{s}\right)$. More generally $\mathcal{L}_{\mathrm{DBI}}(F+$ $\left.b^{\varepsilon}, \phi, g^{\varepsilon}, g_{s}\right)$ admits the first order equations (71) that using tensor notation read

$$
\mathcal{F}_{\mu \nu} \equiv F_{\mu \nu}+b^{\varepsilon}{ }_{\mu \nu}=-\sqrt{g} \epsilon_{\mu \nu \rho 0} g^{\rho \sigma} \frac{\partial \phi}{\partial x^{\rho}}+b^{\varepsilon}{ }_{\mu \nu}-b_{\mu \nu}
$$

In the orthonormal reference system $x^{\prime \prime}$, recall diagram (55), equations (89) read

$$
\begin{aligned}
\mathcal{B}_{2}^{\prime \prime} & =-\gamma \partial_{2}^{\prime \prime} \phi^{\prime \prime} \\
\mathcal{B}_{q}^{\prime \prime} & =-\gamma^{-1} \partial_{q}^{\prime \prime} \phi^{\prime \prime} \\
\mathcal{E}_{2}^{\prime \prime} & =e^{\prime \prime}, \quad \mathcal{E}_{q}^{\prime \prime}=0
\end{aligned} \quad q=1,3
$$

with $\partial^{i} \mathcal{B}_{i}^{\prime \prime}=0$ and $\gamma^{-1}=\sqrt{1-\alpha^{\prime 2} e^{\prime \prime 2}}$. In order to obtain (90) from (89) multiply (89) by $\Gamma^{\varepsilon^{T}}$ and by $\Gamma^{\varepsilon}$ and then express all the remaining tensors in terms of $e^{\prime \prime}$ and $b^{\prime \prime}$ [cf. (47), (52)]. Equations (90) are of BPS type, they are first order equations in the gauge potential and the scalar field. They correspond to the BPS equations of noncommutative gauge theory (69). Because of the commutativity of the diagram (55) they can also be obtained from the BPS equations in the $x^{\prime}$ reference system

$$
\mathcal{B}_{i}^{\prime}=-\partial_{i}^{\prime} \phi^{\prime} \quad, \quad \mathcal{E}_{i}^{\prime}=0
$$

using the boost (60) in the $\psi^{\prime}, x_{2}^{\prime}$ plane. [Is is straighforward to explicitly derive (90) from (91) using (61),(63),(65)]. Since (91) is equivalent to (72) (with primed 
variables) then, recalling (62) and (63), we have that (90) is equivalent to

$$
\mathcal{H}_{i}^{\prime \prime}=-\partial_{i}^{\prime \prime} \phi^{\prime \prime}, \quad\left(\partial_{1}^{\prime \prime 2}+\gamma^{2} \partial_{2}^{\prime \prime 2}+\partial_{3}^{\prime \prime 2}\right) \phi^{\prime \prime}=0, \quad \mathcal{E}_{2}^{\prime \prime}=e^{\prime \prime}, \quad \mathcal{E}_{1}^{\prime \prime}=\mathcal{E}_{3}^{\prime \prime}=0
$$

The boost (60) that relates (91) to (90) is a symmetry of the action $\int d^{3} x \mathcal{L}_{\text {DBI }}$. Indeed under (60), for a generic static configuration, the functional $\Sigma_{\breve{H}}$ [cf. (59)] is invariant and from (80) where now $J_{i}{ }^{j}=\frac{\partial x^{\prime j}}{\partial x^{\prime \prime i}}$ we have, as in (81), (84)

$$
\begin{aligned}
\partial_{i}^{\prime} \mathcal{B}^{\prime i} d^{3} x^{\prime} & =\partial_{i}^{\prime \prime} \mathcal{B}^{\prime \prime i} d^{3} x^{\prime \prime} \\
\mathcal{B}^{\prime} \mathcal{H}_{i}^{\prime} d^{3} x^{\prime} & =\mathcal{B}^{\prime \prime}{ }^{i} \mathcal{H}_{i}^{\prime \prime} d^{3} x^{\prime \prime}
\end{aligned}
$$

recalling (56) we then have

$$
\mathcal{L}\left(\mathcal{E}^{\prime}, \mathcal{B}^{\prime}, \phi^{\prime}\right) d^{3} x^{\prime}=\mathcal{L}\left(\mathcal{E}^{\prime \prime}, \mathcal{B}^{\prime \prime}, \phi^{\prime \prime}\right) d^{3} x^{\prime \prime}
$$

Also the canonical energy $\int d^{3} x^{\prime} H_{c}=\int d^{3} x^{\prime} T_{c}^{00}$, obtained from the canonical energy momentum tensor $T_{c}^{\mu \nu}=\frac{\partial \mathcal{L}\left(\mathcal{A}^{\prime}, \phi^{\prime}\right)}{\partial \partial_{\mu} \mathcal{A}_{\rho}^{\prime}} \partial^{\nu} \mathcal{A}_{\rho}^{\prime}+\eta^{\mu \nu} \mathcal{L}$ is invariant under the boost (60), indeed for a generic static configuration we have $H_{c}=-\mathcal{L}$. As (93) shows, the magnetic charge too is invariant under the boost (60).

Given a BPS state (91), the boosted one (90) has a charge

$$
Z_{m}^{\prime \prime}=\int d^{3} x^{\prime \prime} \mathcal{B}^{\prime \prime} \partial_{i} \phi^{\prime \prime}=-\int d^{3} x^{\prime \prime} \mathcal{B}^{\prime \prime}{ }^{i} \mathcal{H}_{i}^{\prime \prime}=-\int d^{3} x^{\prime} \mathcal{B}^{i} \mathcal{H}_{i}^{\prime}=\int d^{3} x^{\prime} \mathcal{B}^{\prime} \partial_{i} \phi^{\prime}=Z_{m}^{\prime}
$$

and, recalling (87), a gravitational energy

$$
\Sigma^{\prime \prime}=\int d^{3} x^{\prime \prime} H\left(\mathcal{D}^{\prime \prime}, \mathcal{B}^{\prime \prime}, \phi^{\prime \prime}\right)=\Sigma^{\prime}+\frac{1}{g_{s}} \int d^{3} x^{\prime \prime} \mathcal{E}_{i}^{\prime \prime} \mathcal{D}^{\prime \prime i}
$$

We see that the energy is of BPS type, indeed it is the sum of the old BPS energy $\Sigma^{\prime}$ plus the topological charge $Z_{e}^{\prime \prime}=\frac{1}{g_{s}} \int d^{3} x^{\prime \prime} \mathcal{E}_{i}^{\prime \prime} \mathcal{D}^{\prime \prime i}=-\frac{1}{g_{s}} \int d^{3} x^{\prime \prime} \partial_{i} \psi^{\prime \prime} \mathcal{D}^{\prime \prime}{ }^{i}$. The explicit value of $\mathcal{D}^{\prime \prime i}$ [see definition (83)] can be obtained from (95), (63), (65) expressing $\mathcal{D}^{\prime \prime i}$ in terms of $\partial_{i}^{\prime} \phi^{\prime}$. We have

$$
\mathcal{D}^{\prime \prime 2}=e^{\prime \prime} \gamma, \quad \mathcal{D}^{\prime \prime 1}=\mathcal{D}^{\prime \prime 3}=0
$$

At second order in $e^{\prime \prime}$ the energy is $\Sigma^{\prime \prime}=\frac{1}{\alpha^{\prime 2} g_{s}} \int d^{3} x^{\prime \prime}+\left|Z_{m}^{\prime \prime}\right|+\frac{e^{\prime \prime 2}}{2 g_{s}} \int d^{3} x^{\prime \prime}$, we recognize the brane tension, the topological charge and the extra tension due to the energy of the electric field $e^{\prime \prime}$. The energy $\Sigma^{\prime \prime}$ to all orders in $e^{\prime \prime}$ is obtained just replacing in the last addend $\frac{1}{2} e^{\prime \prime 2}$ with the relativistic term $\gamma-1$.

Solution (73) in the $x^{\prime \prime}$ reference system reads ${ }^{\mathrm{f}}$

$$
\phi^{\prime \prime}=-\gamma b^{\prime \prime} x^{\prime \prime 3}-\frac{1}{2 R}
$$

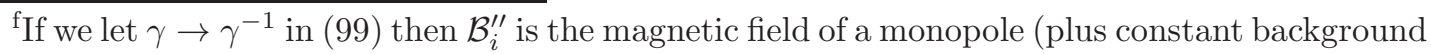
$\left.b^{\prime}\right)$ moving with velocity $\beta$ in the $x_{2}^{\prime \prime}$ direction
} 


$$
\begin{aligned}
\mathcal{B}^{\prime \prime} i & =-\frac{1}{2 \gamma} \frac{x^{\prime \prime i}}{R^{3}}+b^{\prime \prime} \delta^{i 3} \\
\mathcal{E}_{2}^{\prime \prime} & =e^{\prime \prime}, \quad \mathcal{E}_{1}^{\prime \prime}=\mathcal{E}_{3}^{\prime \prime}=0 \quad, \quad R^{2} \equiv\left(x^{\prime \prime 1}\right)^{2}+\gamma^{-2}\left(x^{\prime \prime 2}\right)^{2}+\left(x^{\prime \prime 3}\right)^{2}
\end{aligned}
$$

In the next subsubsection we show that the string tension associated to this configuration is that of a D1-string, as we expect from a BPS state. Notice that the shape of the funnel representing the string is no more symmetric in the $x_{1}^{\prime \prime}, x_{2}^{\prime \prime}$ directions. A section determined by $\alpha^{\prime} \phi^{\prime \prime}+\alpha^{\prime} \gamma b^{\prime \prime} x_{3}^{\prime \prime}=$ const is an ellipsis in the $x_{2}^{\prime \prime}, \rho$ plane, here $\rho=\sqrt{x_{1}^{\prime \prime 2}+x_{3}^{\prime \prime 2}}$. The ratio between the ellipsis axes is given by $\gamma=\frac{1}{\sqrt{1-\alpha^{\prime 2} e^{\prime \prime 2}}}=\sqrt{\frac{\alpha^{\prime 2}+\theta^{2}}{\alpha^{\prime 2}+\theta^{2}-\varepsilon^{2}}}$

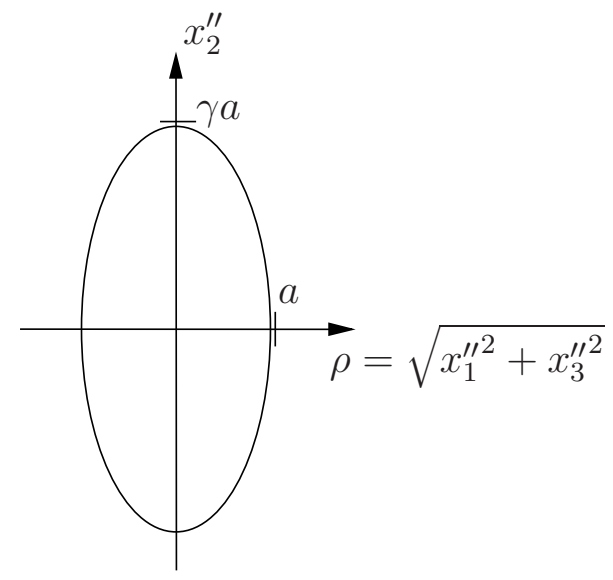

Since $\gamma b^{\prime \prime}=\theta / \alpha^{\prime 2}$, when $\alpha^{\prime} \rightarrow 0$ with open string parameters held fixed the branestring angle goes to zero: the brane is now perpendicular to the $x_{3}^{\prime \prime}$ axis and the string lies on the brane. The ellipsis (100) (now with $x_{3}^{\prime \prime}=0$ ) has the axis $a=\frac{-\alpha^{\prime}}{2 \text { const }}$ and therefore the string is eventually localized at $x_{1}^{\prime \prime}=0$ for $\alpha^{\prime} \rightarrow 0$ (this is the same as in the commutative case $\theta=\varepsilon=0$ ). On the other hand, since for $\theta^{\varepsilon}$ light-like $\gamma \sim \theta / \alpha^{\prime}$, the other ellipsis axis is $\gamma a=\frac{-\theta}{2 \text { const }}$ so that the string smoothly opens into the brane along the $x_{2}^{\prime \prime}$ direction independently from $\alpha^{\prime} \rightarrow 0$.

\subsubsection{String Tension}

In this subsection we match the noncommutative string tension (88) with the string tension of configuration (99) written in the $x$ reference system, i.e. of (73) with $\mathcal{E}_{2}=-b^{\varepsilon}{ }_{02}, \mathcal{E}_{1}=\mathcal{E}_{2}=0$. This last is a solution of the $\mathcal{L}_{\mathrm{DBI}}\left(F+b^{\varepsilon}, \phi, g^{\varepsilon}, g_{s}\right)$ first order equations (89). We begin by reviewing the string tension in the $x^{\prime}$ reference system, i.e. for configuration (99) with $\varepsilon=0$. Let $\alpha^{\prime} \Phi^{\prime}$ be the axis perpendicular to the brane, it has an angle $\zeta$ with the string axis $\alpha^{\prime} \phi^{\prime} ; \tan \zeta=\alpha^{\prime} b^{\prime}=\theta / \alpha^{\prime}$. We have (see Fig. 2, with axes $\bar{x}^{3}, \alpha^{\prime} \phi, \alpha^{\prime} \bar{\phi}$ renamed $x_{3}^{\prime}, \alpha^{\prime} \Phi^{\prime}, \alpha^{\prime} \phi^{\prime}$ )

$$
\alpha^{\prime} \Phi^{\prime}=\cos \zeta \alpha^{\prime} \phi^{\prime}+\sin \zeta x_{3}^{\prime}=-\alpha^{\prime} \cos \zeta \frac{1}{2 r^{\prime}}
$$


where $r^{\prime 2}=x_{1}^{\prime 2}+x_{2}^{\prime 2}+x_{3}^{\prime 2}$. The string tension $T^{\prime}$ is

$$
T^{\prime} \frac{\Delta \alpha^{\prime} \Phi^{\prime}}{\cos \zeta}=\Delta \Sigma^{\prime}
$$

where $\Delta \Sigma^{\prime}=\left.\Sigma^{\prime}\right|_{\alpha^{\prime} \Phi^{\prime}} ^{\alpha^{\prime}{ }^{\prime}+\Delta \alpha^{\prime} \Phi^{\prime}}$. We have

$$
\begin{aligned}
\Delta_{0} \Sigma^{\prime} & =\left.\Sigma^{\prime}\right|_{\alpha^{\prime} \Phi_{0}^{\prime}} ^{\alpha^{\prime}{ }^{\prime}+\Delta \alpha^{\prime} \Phi_{0}^{\prime}}=\frac{1}{g_{s}} \int_{V_{0}} d^{3} x^{\prime}\left(\nabla^{\prime} \phi^{\prime}\right)^{2} \\
& =\frac{1}{g_{s} \cos ^{2} \zeta} \int_{V_{0}} d^{3} x^{\prime}\left(\nabla^{\prime} \Phi^{\prime}\right)^{2}+\frac{1}{g_{s}} \int_{V_{0}} d^{3} x^{\prime} b^{2} \\
& =\frac{1}{g_{s} \cos ^{2} \zeta} \int_{\partial V_{0}} d S^{\prime} \Phi^{\prime} \nabla^{\prime} \Phi^{\prime}+\frac{1}{g_{s}} V_{0} b^{\prime 2} \\
& =\frac{2 \pi}{g_{s} \cos \zeta} \Delta \Phi_{0}^{\prime}+\frac{1}{g_{s}} V_{0} b^{\prime 2}
\end{aligned}
$$

where $V_{0}$ is the region in space such that $\Phi^{\prime}$ is between $\Phi_{0}^{\prime}$ and $\Phi_{0}^{\prime}+\Delta \Phi_{0}^{\prime}$, and $\partial V_{0}$ is given by the sphere $\Phi^{\prime}=\Phi_{0}^{\prime}+\Delta \Phi_{0}^{\prime}$ and the sphere $\Phi^{\prime}=\Phi_{0}^{\prime}$. In the fourth equality we integrated by parts and used that $\Phi^{\prime}$ is divergence free in $V_{0}$. When $\Phi_{0}^{\prime}>>1$, then $V_{0} \sim 0$ if $\Delta \Phi_{0}^{\prime}$ is fixed, it follows that the energy is simply proportional to $\Delta \Phi_{0}^{\prime}$ and the string tension is that of a D1-string

$$
T^{\prime}=\frac{2 \pi}{g_{s} \alpha^{\prime}}
$$

In order to obtain the string tension associated to the field configuration (99), we recall that $\phi^{\prime \prime}\left(x^{\prime \prime}\right)=\phi^{\prime}\left(x^{\prime}\right)$ and $x_{3}^{\prime \prime}=x_{3}^{\prime}$, so that $\Phi^{\prime \prime}\left(x^{\prime \prime}\right)=\Phi^{\prime}\left(x^{\prime}\right)$. Then from (102), 97), 98) we have

$$
T^{\prime \prime}=T^{\prime}=\frac{2 \pi}{g_{s} \alpha^{\prime}}
$$

Similarly, when $x_{3}^{\prime \prime}>>1$ then $\frac{1}{r^{\prime 4}} \sim 0$, and we also have

$$
\left.\Delta W^{\prime \prime} \equiv \Delta \Sigma^{\prime \prime}\right|_{x_{3}^{\prime \prime}} ^{x_{3}^{\prime \prime}+\Delta x_{3}^{\prime \prime}}=\left.\left.\Delta \Sigma^{\prime}\right|_{x_{3}^{\prime}} ^{x_{3}^{\prime}+\Delta x_{3}^{\prime}} \sim \frac{-1}{g_{s}} \int d^{3} x^{\prime} \frac{b^{\prime} x_{3}^{\prime}}{r^{\prime 3}}\right|_{x_{3}^{\prime}} ^{x_{3}^{\prime}+\Delta x_{3}^{\prime}}=\frac{-2 \pi}{g_{s}} b^{\prime} \Delta x_{3}^{\prime \prime}
$$

$\Delta W^{\prime \prime}$ is a string-brane interaction energy stored in between $x_{3}^{\prime \prime}$ and $x_{3}^{\prime \prime}+\Delta x_{3}^{\prime \prime}$, it is the work necessary to move the charge $-q_{m}=-2 \pi$ from the point $\mathcal{P}$ on the brane to the point $\mathcal{P}+\Delta \mathcal{P}$ on the brane, where the value of the third coordinate of $\mathcal{P}$ and $\mathcal{P}+\Delta \mathcal{P}$ is respectively $x_{3}^{\prime \prime}$ and $x_{3}^{\prime \prime}+\Delta x_{3}^{\prime \prime}$. Since the brane for $x_{3}^{\prime \prime}>>1$ lies in the $\phi^{\prime \prime}+b^{\prime} x_{3}^{\prime \prime}=0$ plane (recall $\gamma b^{\prime \prime}=b^{\prime}$ ), the charge $-q_{m}$ moves a distance $\frac{\Delta x_{3}^{\prime \prime}}{\cos \zeta}$, therefore to $\Delta W^{\prime \prime}$ is associated a tension

$$
t^{\prime \prime}=\frac{\Delta W^{\prime \prime}}{\Delta x_{3}^{\prime \prime}} \cos \zeta
$$


In the $x$ reference system, see (44), we have, from the transformation rule of the energy-momentum vector and from $x_{3}^{\prime \prime}=x_{3}$ and $\phi^{\prime \prime}\left(x^{\prime \prime}\right)=\phi(x)$

$$
\begin{gathered}
\Delta \Sigma=\gamma \Delta \Sigma^{\prime \prime}, \quad \Delta W=\gamma \Delta W^{\prime \prime} \\
T=\gamma T^{\prime \prime}, \quad t=\gamma t^{\prime \prime}
\end{gathered}
$$

As in 29] we project the energy of the string on the brane (we consider the shadow of the string on the brane) and obtain a total tension along the string shadow

$$
\frac{1}{\sin \zeta} T+t=\frac{2 \pi \alpha^{\prime}}{\sqrt{\alpha^{\prime 2}+\theta^{2}-\varepsilon^{2}} \theta g_{s}}=\frac{2 \pi}{G_{s}^{\varepsilon} \theta}
$$

where in the last equality we used (19) and (41). The tension (110) is the same as the noncommutative string tension (88) of Gross-Nekrasov BPS soliton in the presence of $\theta^{\varepsilon}$ noncommutativity. We thus confirm that SW map relates this $\theta^{\varepsilon}$ noncommutative soliton to (99) written in the $x$ reference system.

\subsubsection{Dual string-brane configuration}

We now duality rotate configuration (99) and obtain a soliton solution that describes a fundamental string ending on a D3-brane with electric and magnetic background. Under the rotation (11) with $a=d=0, c=-b^{-1}=2 \pi$ and $S_{1}=C=0, g_{\mu \nu}=\eta_{\mu \nu}$ we have

$$
\left(g_{Y M}^{D}\right)^{2}=g_{s}^{D}=\frac{4 \pi^{2}}{g_{s}} \quad, \quad C_{D}=0 \quad, \quad g_{\mu \nu}^{D}=\frac{2 \pi}{g_{s}} \eta_{\mu \nu} \quad, \quad \phi_{D}^{\prime \prime}=\left(\frac{2 \pi}{g_{s}}\right)^{\frac{1}{2}} \phi^{\prime \prime}
$$

and from $\mathcal{E}_{i}=-\mathcal{F}_{0 i}, \mathcal{B}^{i}=\mathcal{F}^{* 0 i}, \mathcal{D}^{i}=-g_{s} \tilde{\mathcal{K}}^{0 i}, \mathcal{H}_{i}=-g_{s} \sqrt{g} \mathcal{K}_{0 i}$ and (99), (92), (98) we obtain the BPS type equations

$$
\begin{aligned}
\mathcal{D}_{D}^{\prime \prime 2} & =-\sqrt{g^{D}}\left(\frac{2 \pi}{g_{s}^{D}}\right)^{\frac{1}{2}} \gamma \partial^{\prime \prime 2} \phi_{D}^{\prime \prime} \\
\mathcal{D}_{D}^{\prime \prime q} & =-\sqrt{g^{D}}\left(\frac{2 \pi}{g_{s}^{D}}\right)^{\frac{1}{2}} \gamma^{-1} \partial^{\prime \prime q} \phi_{D}^{\prime \prime} \\
\mathcal{H}_{i}^{\prime \prime D} & =\frac{g_{s}^{D}}{2 \pi} \sqrt{g^{D}} e^{\prime \prime} \delta_{i 2}
\end{aligned}
$$

or equivalently

$$
\mathcal{E}_{i}^{\prime \prime D}=-\left(\frac{g_{s}^{D}}{2 \pi}\right)^{\frac{1}{2}} \partial_{i}^{\prime \prime} \phi_{D}, \quad\left(\partial_{1}^{\prime \prime 2}+\gamma^{2} \partial_{2}^{\prime \prime 2}+\partial_{3}^{\prime \prime 2}\right) \phi_{D}^{\prime \prime}=0, \quad \mathcal{B}_{D}^{\prime \prime}=-\frac{2 \pi}{g_{s}^{D}} \gamma e^{\prime \prime} \delta^{i 2}
$$


The dual of solution (99) is given by (111) and

$$
\mathcal{A}_{0}^{\prime \prime D}=-\left(\frac{2 \pi}{g_{s}}\right)^{\frac{1}{2}} \phi_{D}^{\prime \prime} \quad, \quad \mathcal{A}_{1}^{\prime \prime D}=-\frac{2 \pi}{g_{s}} \gamma e^{\prime \prime} x^{\prime \prime 3} \quad, \quad \mathcal{A}_{2}^{\prime \prime D}=\mathcal{A}_{3}^{\prime \prime D}=0
$$

Consider now the $\theta^{\varepsilon}$ light-like case. We then have $\operatorname{det} \Gamma^{\varepsilon}=1$ and duality rotations commute with the coordinate transformation $\Gamma^{\varepsilon}$. They also commute with the $\zeta$-rotation because this rotates $X=\alpha^{\prime} \phi$ with $x^{3}$ and under duality $X$ and $x^{3}$ are unchanged [under duality $\alpha^{\prime} \phi_{D} \neq \alpha^{\prime} \phi$ but $X_{D}=X$ because it is the component $\eta_{X X}$ of the target space metric that changes according to (111)]. In conclusion applying the $\Gamma^{\varepsilon}$ coordinate transformation, the $\zeta$-rotation and SW map we obtain from (115) and (111) the noncommutative fields $\widehat{A}^{D}, \widehat{\phi}_{D}$ with light-like noncommutativity $\theta_{D}^{\varepsilon \mu \nu}=\frac{g_{s}}{2 \pi} \theta^{\varepsilon * \mu \nu}$. These noncommutative fields correspond to a fundamental string ending on a D3-brane with light-like background. The $\widehat{A}^{D}, \widehat{\phi}_{D}$ fields solve also $\widehat{\mathcal{L}}_{\mathrm{DBI}}\left(\widehat{F}, \widehat{\phi}, \star_{\theta_{D}}\right)$ where $\theta_{D}$ is just the space part of $\theta_{D}^{\varepsilon}$, they describe a spacenoncommutative electric monopole with a string attached.

\section{Acknowledgements}

I wish to thank Sergei M. Kuzenko, Yaron Oz, Stefan Theisen, for helpful discussions. I acknowledge fruitful discussions with Branislav Jurčo, Olaf Lechtenfeld, Ivo Sachs and Peter Schupp. I thank Leonardo Castellani for his comments and suggestions. This work has been supported by Alexander von Humboldt-Stiftung.

\section{References}

[1] M. Born and L. Infeld, Proc. Roy. Soc. Lond. A 144 (1934) 425.

[2] E. B. Bogomolny, Sov. J. Nucl. Phys. 24 (1976) 449 [Yad. Fiz. 24 (1976) 861].

[3] M. K. Gaillard and B. Zumino, Nucl. Phys. B 193 (1981) 221. B. Zumino, "Duality rotations", in "Quantum Structure of Space and Time", M. j. Duff and C. J. Isham eds. Cambridge University Press, 1982 p363.

[4] J. H. Schwarz and P. C. West, Phys. Lett. B 126 (1983) 301.

J. H. Schwarz, Nucl. Phys. B 226 (1983) 269.

P. S. Howe and P. C. West, Nucl. Phys. B 238 (1984) 181.

[5] A. A. Tseytlin, Nucl. Phys. B 469 (1996) 51 hep-th/9602064.

[6] G. W. Gibbons and D. A. Rasheed, Phys. Lett. B 365 (1996) 46 hepth/9509141. 
[7] M. K. Gaillard and B. Zumino, "Self-duality in nonlinear electromagnetism," in "Supersymmetry and Quantum Field Theory", J. Wess and V. P. Akulov eds., Springer Verlag, 1998, p. 121 hep-th/9705226.

[8] M. K. Gaillard and B. Zumino, "Nonlinear electromagnetic self-duality and Legendre transformations," in "Duality and Supersymmetric theories", D. I. Olive and P. C. West eds., Cambridge University Press, 1999, p. 33 hepth/9712103.

[9] C. G. Callan and J. M. Maldacena, Nucl. Phys. B 513 (1998) 198 hepth/9708147].

[10] G. W. Gibbons, Nucl. Phys. B 514 (1998) 603 [hep-th/9709027.

[11] J. P. Gauntlett, J. Gomis and P. K. Townsend, JHEP 9801 (1998) 003 hepth/9711205.

[12] A. Connes, M. R. Douglas and A. Schwarz, JHEP 9802 (1998) 003 hepth/9711162.

[13] N. Nekrasov and A. Schwarz, Commun. Math. Phys. 198 (1998) 689 hepth/9802068.

[14] A. Schwarz, Nucl. Phys. B 534 (1998) 720 hep-th/9805034.

P. Ho, Phys. Lett. B 434 (1998) 41 hep-th/9803166.

D. Brace, B. Morariu and B. Zumino, Nucl. Phys. B 545 (1999) 192 hepth/9810099.

[15] C. Chu and P. Ho, Nucl. Phys. B 550 (1999) 151 hep-th/9812219.

[16] K. Hashimoto, JHEP 9907 (1999) 016 hep-th/9905162.

[17] N. Seiberg and E. Witten, JHEP9909 (1999) 032 hep-th/9908142.

[18] R. Gopakumar, S. Minwalla and A. Strominger, JHEP 0005 (2000) 020 hepth/0003160.

[19] J. A. Harvey, P. Kraus, F. Larsen and E. J. Martinec, JHEP 0007 (2000) 042 hep-th/0005031.

[20] N. Seiberg, L. Susskind and N. Toumbas, JHEP0006 (2000) 044 hepth/0005015.

J. L. Barbon and E. Rabinovici, Phys. Lett. B 486 (2000) 202 [hep-th/0005073].

J. Gomis and T. Mehen, Nucl. Phys. B 591 (2000) 265 hep-th/0005129. 
[21] O. J. Ganor, G. Rajesh and S. Sethi, Phys. Rev. D 62 (2000) 125008 hepth/0005046.

[22] R. Gopakumar, J. Maldacena, S. Minwalla and A. Strominger, JHEP0006 (2000) 036 hep-th/0005048.

[23] O. Aharony, J. Gomis and T. Mehen, JHEP0009 (2000) 023 [hep-th/0006236.

[24] S. Rey and R. von Unge, Phys. Lett. B 499 (2001) 215 hep-th/0007089.

[25] J. X. Lu, S. Roy and H. Singh, Nucl. Phys. B 595 (2001) 298 hep-th/0007168.

[26] M. Alishahiha, Y. Oz and J. G. Russo, JHEP 0009 (2000) 002 hepth/0007215.

[27] T. Kimura and I. Oda, Int. J. Mod. Phys. A 16 (2001) 503 hep-th/9904019.

[28] S. M. Kuzenko and S. Theisen, Fortsch. Phys. 49 (2001) 273 hep-th/0007231.

[29] D. J. Gross and N. A. Nekrasov, JHEP 0007 (2000) 034 hep-th/0005204.

[30] D. J. Gross and N. A. Nekrasov, JHEP 0010 (2000) 021 hep-th/0007204.

[31] A. Hashimoto and K. Hashimoto, JHEP 9911 (1999) 005 hep-th/9909202

K. Hashimoto and T. Hirayama, Nucl. Phys. B 587 (2000) 207 hepth/0002090.

[32] D. Mateos, Nucl. Phys. B 577 (2000) 139 hep-th/0002020.

[33] S. Moriyama, Phys. Lett. B 485 (2000) 278 [hep-th/0003231.

[34] S. Moriyama, JHEP 0008 (2000) 014 hep-th/0006056.

[35] K. Hashimoto, T. Hirayama and S. Moriyama, JHEP 0011 (2000) 014 hepth/0010026.

[36] K. Hashimoto, JHEP 0012 (2000) 023 hep-th/0010251.

[37] J. Gomis, K. Kamimura and J. Llosa, Phys. Rev. D 63 (2001) 045003 hepth/0006235.

J. Gomis, K. Kamimura and T. Mateos, JHEP 0103 (2001) 010 hepth/0009158.

[38] D. Bak and K. Lee, Phys. Lett. B 495 (2000) 231 hep-th/0007107.

[39] D. Bak, S. U., K. Lee and J. Park, Phys. Rev. D 63 (2001) 125010 hepth/0011099. 
[40] B. Jurco, P. Schupp and J. Wess, Nucl. Phys. B 604 (2001) 148 hepth/0102129.

[41] O. Lechtenfeld, A. D. Popov and B. Spendig, JHEP 0106 (2001) 011 hepth/0103196.

O. Lechtenfeld and A. D. Popov, "Noncommutative Multi-Solitons in 2+1 Dimensions," hep-th/0106213.

[42] P. Aschieri, Mod. Phys. Lett. A 16 (2001) 163 [hep-th/0103150.

[43] M. R. Garousi, "Dirac-Born-Infeld action, Seiberg-Witten map, and Wilson lines," hep-th/0105139.

[44] K. Hashimoto and H. Ooguri, "Seiberg-Witten transforms of noncommutative solitons," hep-th/0105311. 